\title{
Reconstrucción paleohidrológica de la Salina de Ambargasta (Argentina) durante los últimos 45000 años mediante geoquímica de isótopos estables
}

\author{
Gabriela A. Zanor, Eduardo L. Piovano, Daniel Ariztegui, Torsten Vennemann, \\ Oscar A. Martínez-Jaime, María Jesús Puy-Alquiza
}

Gabriela A. Zanor

gzanor@ugto.mx

Oscar A. Martínez-Jaime

División de Ciencias de la Vida (DICIVA), Campus Irapuato-Salamanca, Universidad de Guanajuato, Carretera Irapuato-Silao Km 9, C.P. 36500, Irapuato, México.

\section{Eduardo L. Piovano}

Centro de Investigaciones en Ciencias de la Tierra (CICTERRA)-CONICET, Universidad Nacional de Córdoba, Av. Velez Sarsfield 1611, X5016GCA Córdoba, Argentina.

\section{Daniel Ariztegui}

Section des Sciences de la Terre et de l'environnement, Université de Genève, Rue des Maraichers 13, 1205 Ginebra, Suiza.

\section{Torsten Vennemann}

Faculté des géosciences et de l'environnement, Université de Lausanne, Chavannes-près-Renens 1022, 1015 Lausanne, Suiza.

\section{María Jesús Puy-Alquiza}

Departamento de Minas, Metalurgia y Geología, Campus Guanajuato, Universidad de Guanajuato, Ex. Hda. de San Matías s/n, Fracc. San Javier, C.P. 36025, Guanajuato, México.

BOL. SOC. GEOL. MEX. 2017 VOL. 69 NO. 3

\section{RESUMEN}

Salina de Ambargasta es un sistema plava ubicado en la región subtropical de Sudamérica $\left(29{ }^{\circ} \mathrm{S}\right)$, influenciado por el Sistema Monzónico Sudamericano. Se analizó el registro isotópico de $\delta^{13} \mathrm{C}$ y $\delta^{18} \mathrm{O}$ en carbonatos a lo largo de dos núcleos sedimentarios fechados previamente por ${ }^{14} \mathrm{C}$ AMS, a fin de identificar los cambios hidrológicos desde los ca. 45000 años cal. AP hasta la actualidad. Adicionalmente, se calcularon los coeficientes de correlación de Pearson entre los isótopos estables y entre cada isótopo con los valore de Pérdida por Ignición $\left(\mathrm{PPI}_{550}\right.$ y $\left.\mathrm{PPI}_{1000}\right)$ en los sedimentos. Posterior a un intervalo seco documentado a los $c a .45000$ años cal. AP, los valores de $\delta^{13} \mathrm{C}$ más negativos (promedio $=-5.10 \%$ ) evidenciaron un periodo más húmedo entre los ca. 39600 y 23600 años cal. AP (MIS 3), que contribuvó al desarrollo de lagunas perennes carbonatadas y efímeras sulfatadas. Por su parte, los valores de $\delta^{18} \mathrm{O}$ fueron más positivo (promedio $=-2.76$ y máximo $=4.86 \%$ ) sugiriendo periodos sujetos a altas tasas de evaporación. Desde los ca. 23600 años cal. AP al Presente (MIS 2 y MIS 1), los sedimentos presentaron composiciones promedio de $\delta^{13} \mathrm{G}_{\text {carb }}$ relativamente más positivas $(-4.50$ $\%$ ) y valores promedio de $\delta^{18} \mathrm{O}$ comparativamente más negativos $(-4.35 \%$ o) reflejando un aporte menor de agua al sistema y una evaporación más baja, respectivamente. A partir de este registro se consideró el inicio del Último Máximo Glacial (UGM) en Ambargasta, definido en el sur de Sudamérica entre los ca. 25000 y 18000 años AP. En la secuencia de la laguna perenne carbonatada (ca. $39600-26700$ años cal. AP) la existencia de un sistema lacustre hidroló gicamente cerrado fue evidenciado por la correlación entre $\delta^{13} \mathrm{C} \quad$ y $\delta^{18} \mathrm{O} \quad(\mathrm{r}=0.62)$. Las correlacione entre PPI ${ }_{550}$ y PPI ${ }_{1000}$ positivas (laguna carbonatada $\mathrm{r}=0.88 ; c a .39600-26700$ años cal. AP) y negativas lagunas sulfatadas; $\mathrm{r}$ promedio $=-0.81 ; c a .39600$ 23600 años cal. AP) indicaron altas productividades asociadas a la precipitación de evaporitas durante etapas de concentración evaporítica (excursiones positivas en las relaciones isotópicas). Esta investigación permitió inferir que entre los ca. 39600 y 23600 años cal. AP existió una etapa más húmeda y cálida con respecto al periodo $c a .23600$ años cal. AP-Presente en la región de Ambargasta (Argentina central). Este trabajo documentó cambios importantes en la relación Precipitación/Evaporación a lo largo del Pleistoceno tardío y Holoceno, y aportó información clave sobre ventanas de tiempo poco exploradas en el sureste de Sudamérica, que en conjunto permitirán definir patrones regionales de la variabilidad paleoclimática durante el Cuaternario.

Palabras clave: salina, isótopos estables, carbonatos, variabilidad paleoclimática, Pleistoceno tardío, Último Máximo Glacial.

\section{ABSTRACT}

Salina de Ambargasta is a playa system located at the subtropical region in South America $\left(29^{\circ} \mathrm{S}\right)$, and influenced by the South American Monsoon System. The present work analyzed the isotopic records of $\delta^{13} \mathrm{C}$ and $\delta^{18} \mathrm{O}$ in carbonates throughout two sedimentary cores, previously dated by ${ }^{4} C A M S$, in order to identify hydrological changes from ca. 45000 years cal. BP to the present. Additionally, the Pearson correlation coefficients were inspected among the stable isotopes, and also between each isotope and the Loss on Ignition (LOI and $\mathrm{LOI}$ ) values in the sediments. Following a dry phase at ca. 45000 years cal. BP, the most negative isotopic values of $\delta^{13} \mathrm{C}$ (average: $-5.10 \%$ ) reflected a more humid period between ca. 39600 and 23600 years cal. BP (MIS 3) that favored water inflow into perennial carbonate and ephemeral sulfate lakes. Values of $\delta^{18} \mathrm{O}_{\text {carb }}$ were more positive (average: 2.76 and maximum: $4.86 \%$ ) suggesting periods influenced by high evaporation rates. From ca. 23600 years cal. BP to the present (MIS 2 and MIS 1), average compositions of $\delta^{13} \mathrm{C}$ were relatively more positive $(-4.50 \%)$, and $\delta^{I 8} \mathrm{O}_{\text {carb }}$ average values were comparatively more negative $(-4.35 \%$ ), indicating less water input to the system and lower evaporation, respectively. From these records, the beginning of the Last Glacial Maximum (LGM) in Ambargasta is dated between ca. 25000 and 18000 years cal. BP in southern South America. In the sequence of the carbonate perennial lake, the existence of a hydrologically closed lacustrine system (ca. 39600 - 26700 years cal. BP) was revealed by a correlation between $\delta^{13} C_{c a b b}$ and $\delta^{18} O_{c a b h}(r=0.62)$. Positive correlations between $P P I_{550}$ and $P P I_{1000}$ (carbonate lake; $r$ $=0.88 ; \mathrm{ca} .39600-26,700$ years cal. BP) and negative (sulfate lakes; average $r=-0.81$; ca. $39600-23600$ years cal. BP) indicated high productivities associated with precipitation of evaporites during evaporative concentration stages (isotope positive excursions). This research allows inferring that between ca. $39600-23600$ years cal. BP, a more humid and warmer stage occurred with respect to the period between ca. 23600 years cal. BP and the present in the Ambargasta region (central Argentina). This work documents important changes in the Precipitation/Evaporation ratio throughout the Late Pleistocene and Holocene, and provides vital information about time windows practically unexplored in southern South America. Taken together, these allow defining regional patterns of paleoclimatic variability during the Quaternary.

Keyzords: salina, stable isotopes, carbonates, Paleoclimatic variability, Late Pleistocene, Last Glacial Maximum. 


\section{Introducción}

El análisis del registro sedimentario de cuencas hidrológicamente abiertas o cerradas mediante el uso de indicadores o proxies ambientales permite obtener información sobre los cambios climáticos y tectónicos del pasado (Jones y Deocampo, 2003). Dentro del amplio grupo de indicadores ambientales, las relaciones entre los isótopos estables de $\mathrm{C}$ y $\mathrm{O}\left(\delta^{13} \mathrm{C}\right.$ y $\left.\delta^{18} \mathrm{O}\right)$ en sedimentos lacustres, son una herramienta fundamental en los estudios paleohidrológicos ya que poseen un gran potencial como amplificadores de la información hidroclimática almacenada (Talbot, 1990; Chivas et al., 1993; Valero-Garcés et al., 2003; Leng et al., 2005; Liu et al., 2009; Steinman y Abbott, 2013; Costa et al., 2014). Las reconstrucciones paleolimnológicas basadas en la geoquímica de los isótopos estables comúnmente utilizan los carbonatos autigénicos ya que precipitan en equilibrio isotópico con el cuerpo de agua (Cohen, 2003). Según McKenzie y Hollander (1993), en sistemas lacustres abiertos, la precipitación carbonática está influenciada preferentemente por incrementos en el $\mathrm{pH}$ y por la concentración del ión carbonato asociado a la extracción del $\mathrm{CO}_{2}$ por organismos fotosintéticos. En cambio, en sistemas lacustres cerrados y especialmente en climas áridos, los carbonatos precipitan principalmente bajo la influencia de un balance hídrico negativo [Evaporación $(\mathrm{E})>$ Precipitación $(\mathrm{P})$ ], donde los aumentos en las composiciones de $\delta^{13} \mathrm{C}$ y $\delta^{18} \mathrm{O}$ se relacionan con el incremento en la tasa de evaporación (Leng et al., 2005). Con base en lo propuesto por Leng y Marshall (2004), la relación isotópica del $\mathrm{O}$ en los minerales carbonáticos en sistemas lacustres $\left(\delta^{18} \mathrm{O}_{\text {carb }}\right)$ brinda información clave acerca de las paleotemperaturas y de la composición isotópica del $\mathrm{O}$ del cuerpo de agua. Por su parte, los cambios en el $\delta^{13} \mathrm{C}$ de la fase mineral carbonática $\left(\delta^{13} \mathrm{C}_{\text {carr }}\right)$ reflejan las variaciones en el reservorio del Carbono Inorgánico Disuelto Total (CIDT; Talbot y Kelts, 1990) en el cuerpo de agua. En este caso, la respuesta isotópica del C está controlada por tres procesos fundamentales: la composición isotópica de los aportes acuosos, el intercambio de $\mathrm{CO}_{2}$ entre agua-atmósfera y los procesos de fotosíntesis/respiración de la biota dentro del lago.

Li y Ku (1997) utilizaron la correlación entre $\delta^{13} \mathrm{G}_{\text {carb }}$ y $\delta^{18} \mathrm{O}_{\text {carb }}$ como un indicador para reconocer si las cuencas lacustres se comportaron como hidrológicamente cerradas o abiertas a lo largo de su historia evolutiva. Los sistemas cerrados generalmente son los que presentan una covarianza alta entre $\delta^{13} \mathrm{C}$ y $\delta^{18} \mathrm{O}$ ya que estos ambientes experimentan un crecimiento rápido de volumen seguido por una decrecimiento al estar sometidos a una alta evaporación, dando lugar a relaciones más positivas de $\delta^{13} \mathrm{C}$ y $\delta^{18} \mathrm{O}$ (por ej., Piovano et al., 2004). Por el contrario, en las cuencas lacustres abiertas donde el volumen permanece estable o sufre cambios mínimos, la tendencia es mantener sin variaciones abruptas los valores de los isótopos, dando como resultado una covarianza débil o nula entre $\delta^{18} \mathrm{O}$ y $\delta^{13} \mathrm{G}$.

La Salina de Ambargasta se localiza en la región central de Argentina (29 LS y $64^{\circ}$ LO; Figura 1), bajo la influencia del Sistema Monzónico Sudamericano (SMS; Zhou y Lau, 1998; Figura 2a). Un estudio paleoambiental previo realizado en Ambargasta por Zanor et al. (2013) usando indicadores sedimentológicos evidenció una gran fluctuación de ambientes desde el Pleistoceno tardío al presente, abarcando desde lagunas con precipitación carbonatada y yesífera, planicies fangosas salinas hasta planicies fangosas capilares. Recientemente, diferentes investigaciones limnogeológicas mediante el uso de multi-indicadores ambientales y cronologías de alta frecuencia han revelado datos cruciales acerca de la dinámica pasada del SMS al este de la Diagonal Árida (DA; Bruniard, 1982; Figura 2a) en Sudamérica (Piovano et al., 2004; Piovano et al., 2009; Stutz et al., 2010; Córdoba et al., 2015; Guerra et al., 2015). Sin embargo, hasta el momento existen escasos estudios en la región que abarquen ventanas de tiempo más extensas (i.e. Pleistoceno tardío), a través del análisis de proxies sedimentológicas, geoquímicas o biológicas (Kröhling et al., 1999; Prado y Alberdi, 1999; Tonni et al., 1999; Zárate, 2003; 


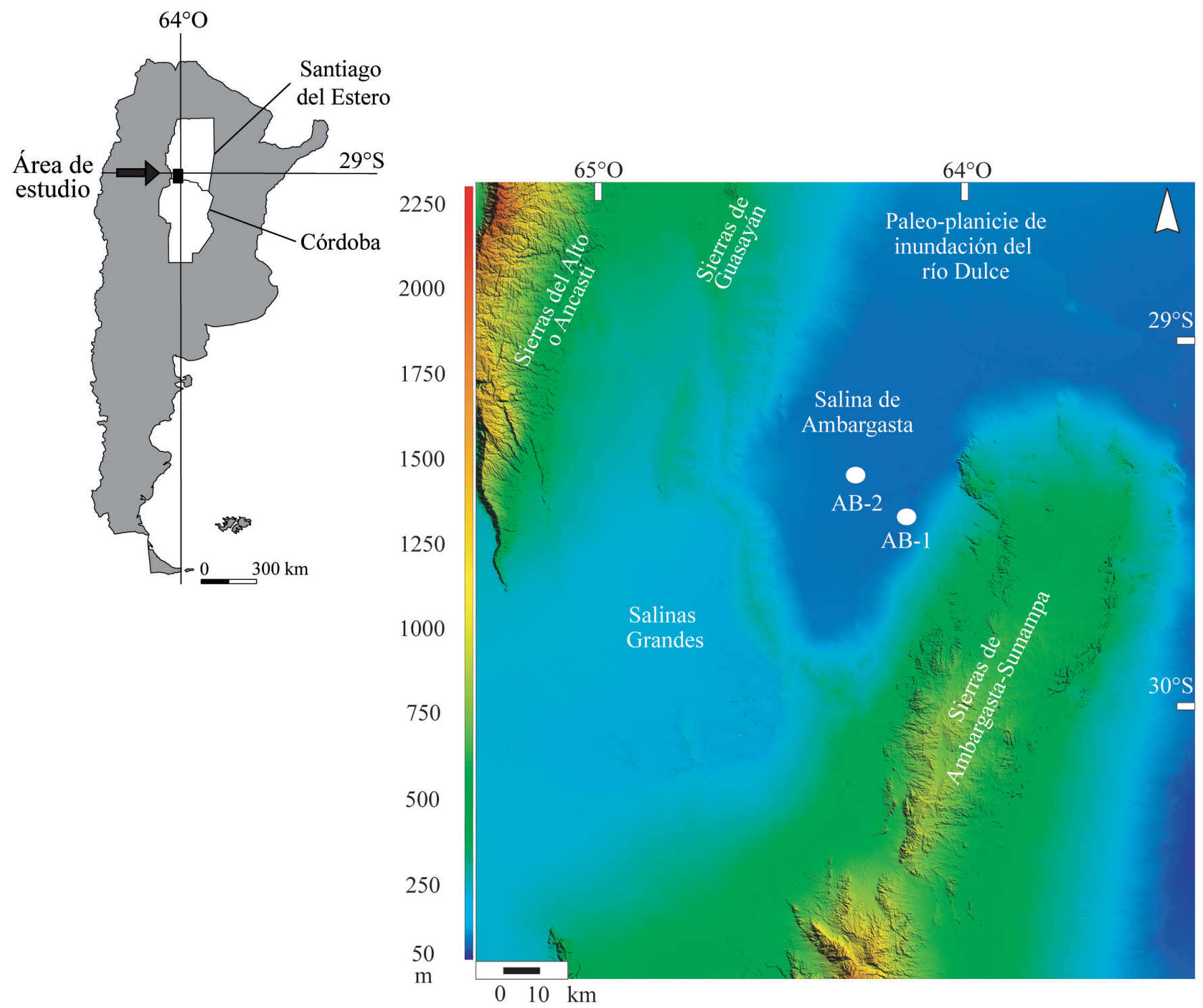

Figura 1 Modelo de Elevación Digital (MED) mostrando los núcleos muestreados (AB-1 y AB-2). Las sierras que rodean al sistema salina de Ambargasta se indican en el MED (todas pertenecientes al sistema montañoso de Sierras Pampeanas) junto con los cuerpos salinos que conforman la Cuenca Saliniana.

Quattrocchio et al., 2008; Blasi et al., 2010, Tonello y Prieto, 2010). Recientemente, Gasparini et al. (2016) presentaron un trabajo donde se recopiló la información existente sobre el estado del conocimiento del Estadio Isotópico Marino 3: MIS 3 (por sus siglas en inglés), en el sur de Sudamérica. Particularmente, Rabassa y Ponce (2013) estudiaron sobre la ocupación poblacional en el continente americano encontrando evidencia de un periodo cálido durante el MIS 3, definido entre los $60000-50000$ y 28000 años cal. AP. Otros estudios en los sectores más australes de Sudamérica (Patagonia argentina) han establecido la cronología del Último Máximo Glacial (UMG; 25000 18000 años AP: dentro del MIS 2; Kull et al., 2003, Kaplan et al., 2008, Rutter et al., 2012) con base en las extensiones máximas de los glaciares. Sin embargo, existe aún mucha incertidumbre sobre la edad de inicio y los cambios ambientales en estos estados del clima durante el Pleistoceno tardío en 
el área del centro de Argentina, y especialmente a partir de registros paleolimnológicos. Es por ello que esta investigación presenta el análisis de isótopos de $\mathrm{C}$ y $\mathrm{O}$ en carbonatos a lo largo del registro sedimentario de Ambargasta con el objetivo de incorporar nuevos indicadores para reconstruir la variabilidad paleohidrológica de baja frecuencia (hasta $c a .45000$ años AP) en las latitudes medias de Argentina. Además, este trabajo explora la relación entre el registro isotópico $\left(\delta^{18} \mathrm{O}_{\text {carb }}\right.$ y $\left.\delta^{13} \mathrm{C}_{\text {carb }}\right)$ y los contenidos de materia orgánica y carbonatos (Pérdida por Ignición; PPI $_{550}$ y PPI $_{1000}$ ) con respecto a las distintas asociaciones de facies, con el fin de fortalecer y refinar las interpretaciones paleoambientales basadas exclusivamente en indicadores sedimentarios (Zanor et al., 2013). El presente estudio contribuye al entendimiento de la dinámica pasada del SMS reconstruyendo fases climáticas e hidrológicas a escalas de tiempo poco exploradas en la región subtropical de Sudamérica.

\section{Geología y clima}

El bolsón salino de Ambargasta, junto con las Salinas Grandes, San Bernardo y La Antigua, forman en conjunto la Cuenca Saliniana (Álvarez et al., 1990; Figura 1). Esta cuenca está situada en la región del antepaís fragmentado de las Sierras Pampeanas (Jordan y Allmendinger et al., 1986), donde los ascensos del basamento están producidos por una deformación tectónica de zócalo. Según Ramos et al. (2002), el ascenso de los bloques de basamento se habría producido alrededor de los últimos 4.5 m.a, dando lugar al desarrollo de encerramientos topográficos y la consecuente formación de cuencas intermontanas (Dávila y Astini, 2003). Los altos de basamento se componen principalmente de metamorfitas de bajo a alto grado intrusionadas por granitos de edades Proterozoicas superiores a Paleozoicas inferiores-medias (Lira et al., 1997). Los depósitos intermontanos de la cuenca incluyen mayormente rocas sedimentarias continentales representadas por conglomerados y areniscas rojizas de edad Terciaria y sedimentos de orígenes aluvial, fluvial, lacustre y eólico del Cuaternario (Lucero, 1979).

La salina de Ambargasta se encuentra ubicada en un sector del continente Sudamericano influenciado por una circulación de tipo monzónica (Vera et al., 2006; Figura 2a). El balance hídrico de Sudamérica está controlado por la superposición de diversos factores tropicales, subtropicales y extratropicales a escala regional (Garreaud et al., 2009; Figura 2a). La Zona de Convergencia Intertropical (ZCIT; Grodsky y Carton, 2003) es un cinturón de bajas presiones caracterizada por el ascenso convectivo de los vientos alisios produciendo nubosidad y precipitación intensa. Durante el invierno austral, las precipitaciones son máximas hacia el norte del Ecuador mientras que en el verano austral la convección se desplaza rápidamente hacia el sur ocasionando precipitaciones fuertes desde la Cuenca del Amazonas hasta el norte de Argentina. Durante el verano austral, los vientos del este son forzados a migrar al sur por la depresión del Chaco (Seluchi y Marengo, 2000), canalizando el flujo de aire entre Los Andes y la Meseta Brasilera a modo de corriente en chorro. La corriente en chorro en capas bajas de Sudamérica (Saulo et al., 2000) transporta grandes cantidades de humedad al sector sureste de América del Sur (Nogués-Paegle y Mo, 1997). Por su parte, la Zona de Convergencia del Atlántico Sur (ZCAS; Liebmann et al., 1999) se conecta preferentemente en la estación de verano con la convección de la ITCZ, produciendo lluvias intensas en la región central del continente. La DA Sudamericana es una banda de precipitación mínima $(<250 \mathrm{~mm} / \mathrm{año})$ que cruza el continente de manera oblicua desde el noreste de Perú hasta la costa Atlántica Patagónica (Figura 2a). Existe una antifase hidrológica desarrollada al $\mathrm{O}$ y al E de la DA, representada por condiciones frías-secas y cálidas-húmedas en las Pampas versus frías-húmedas y cálidas-secas en la Patagonia. En el subtrópico (región Pampeana) las condiciones secas-frías o húmedas-cálidas se corresponden con una disminución o intensificación del régimen 


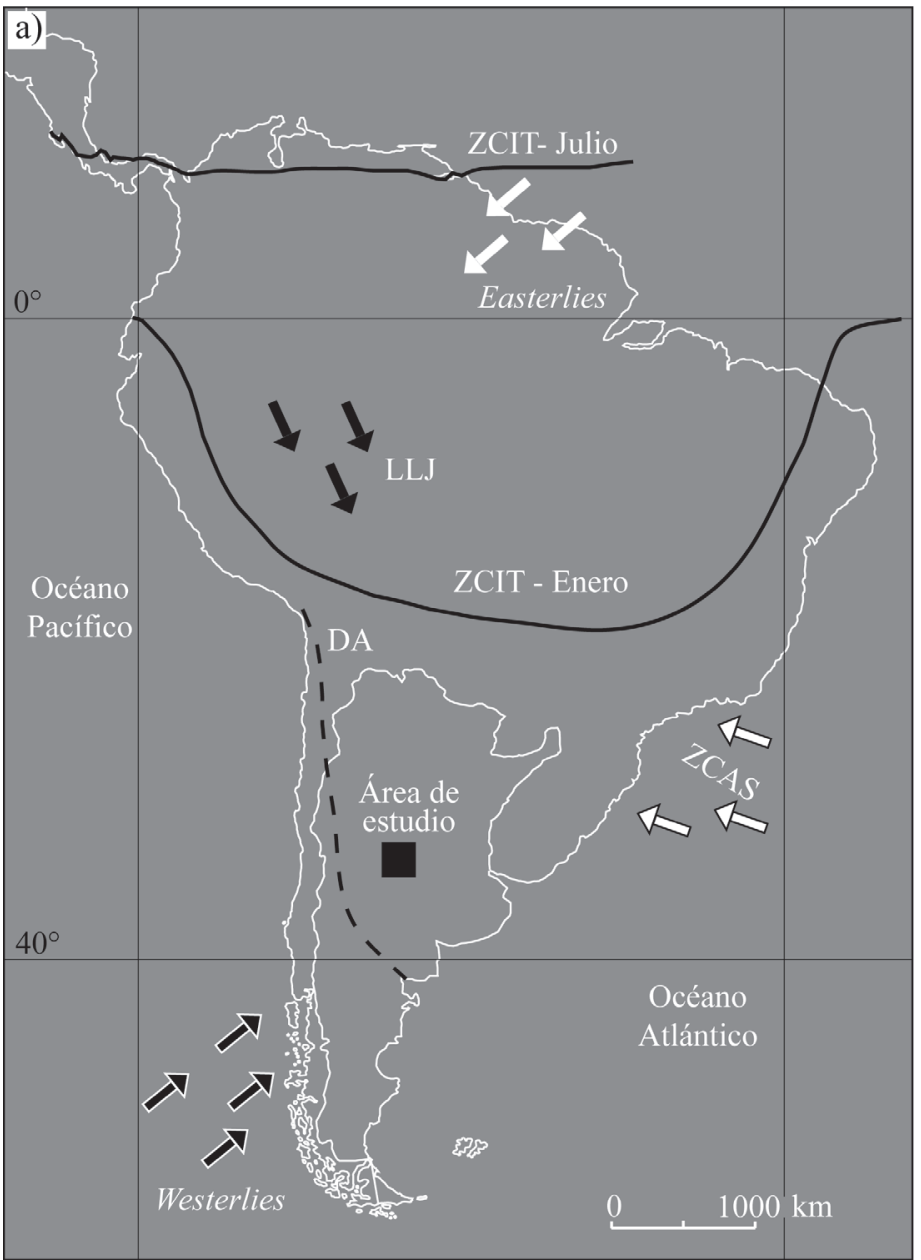

b)

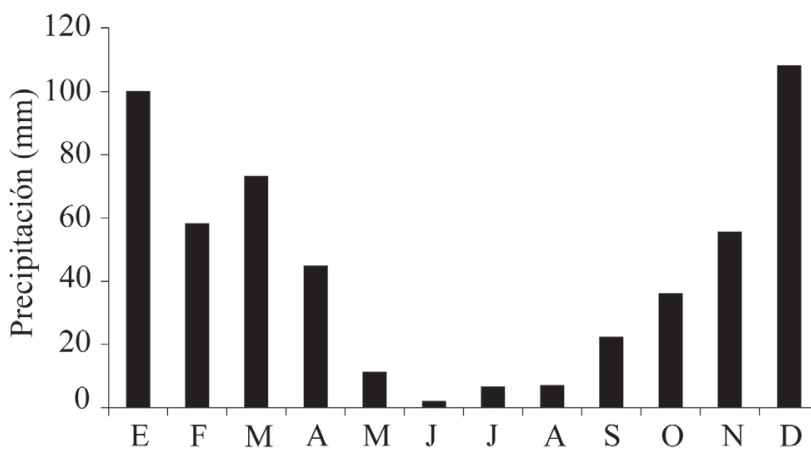

c)

Mes

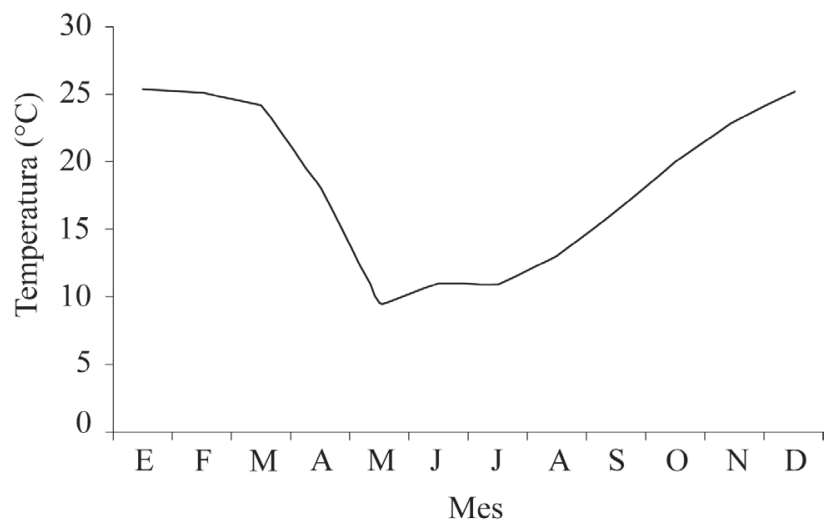

Figura 2 a) Factores del clima que influencian el balance hídrico en Sudamérica. La línea punteada muestra la Diagonal Árida (DA) en Sudamérica. b) Precipitación media mensual histórica en la Salina de Ambargasta (periodo 1986 - 2005). c) Temperatura media mensual histórica en la Salina de Ambargasta (periodo 1986 - 2005).

monzónico Sudamericano, respectivamente (Piovano et al., 2009). En cambio, en la Patagonia las condiciones húmedas-frías se relacionan con un aumento en la actividad de los vientos del oeste australes (Gilli et al., 2005; Mayr et al., 2005).

Particularmente, en la región de Ambargasta la precipitación media anual es de aproximadamente $550 \mathrm{~mm} / \mathrm{año}$, la temperatura media anual es de $19^{\circ} \mathrm{C}$ y la evapotranspiración total anual alcanza los 1300 mm/año (Figuras 2b y 2c). Las máximas temperaturas y precipitaciones ocurren durante el verano (diciembre a marzo) mientras que la estación seca ocurre durante el invierno (junio a septiembre).

\section{Materiales y métodos}

\subsection{ANÁLISIS DE ISÓTOPOS ESTABLES}

Dos núcleos de sedimento (AB-1 y AB-2; Figura 1) fueron obtenidos en septiembre del año 2004 en la Salina de Ambargasta mediante un equipo de sondeo por percusión. AB-1 (longitud: $894 \mathrm{~cm}$, 117 m s.n.m, $29^{\circ} 14^{\prime}$ 'S y $64^{\circ} 13^{\prime} \mathrm{O}$ ) fue extraído en el borde oriental de la salina mientras que AB-2

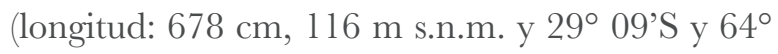
19'O) fue obtenido en el sector central. En este trabajo se presentan los valores de $\mathrm{PPI}_{550}$ y $\mathrm{PPI}_{1000}$ 
determinados mediante la técnica Pérdida por Ignición (PPI; Heiri et al., 2001), previamente publicados en Zanor et al. (2013). Asimismo, la Tabla 1 y la Figura 3 muestran las edades para el registro sedimentario de la Salina de Ambargasta y el modelo cronológico obtenido (Zanor et al., 2013). Las dataciones se realizaron en el Arizona AMS Laboratory, University of Arizona (Tucson, USA), mediante espectrometría de masas con acelerador (AMS).

La determinación de las composiciones de los isótopos de $\mathrm{C}$ y $\mathrm{O}$ en los carbonatos fue realizada en el Institut de Minéralogie et Géochimie, Université de Lausanne (Suiza). Para estos análisis se tomaron muestras cada $10 \mathrm{~cm}$ y en cada cambio litológico. Previo a la medición, se lavaron las muestras con agua destilada para la remoción de

Tabla 1. Edades ${ }^{14} \mathrm{C}$ AMS obtenidas para los sedimentos de los núcleos $A B-1$ y $A B-2$. Las edades fueron calibradas por la curva polinómica de Bard (1998). Material: sedimento orgánico.

\begin{tabular}{|c|c|c|c|c|}
\hline Muestra & $\begin{array}{c}\text { Profundidad } \\
(\mathbf{c m})\end{array}$ & $\begin{array}{c}\text { Edades } \\
\text { radiocarbono }\end{array}$ & Error & $\begin{array}{c}\text { Edad años } \\
\text { cal. AP }\end{array}$ \\
\hline Testigo $A B-1$ & & & & \\
AB-1-724 & 724 & 25790 & \pm 230 & 32506 \\
\hline Testigo $A B-2$ & & & & \\
AB-2-514 & 514 & 21740 & \pm 270 & 25607 \\
\hline AB-2-612 & 612 & 26080 & \pm 830 & 30579 \\
\hline AB-2-638 & 638 & 28690 & \pm 660 & 33514 \\
\hline
\end{tabular}



Figura 3 Modelo cronológico con base en las edades radiocarbónicas obtenidas para el núcleo AB-2 (Zanor et al., 2013). Asumiendo una tasa de sedimentación constante, se realizó una interpolación para inferir las edades de las fases evolutivas de la salina. sales solubles, realizando cinco lavados por muestra. La composición isotópica fue medida a partir del $\mathrm{CO}_{2}$ obtenido de la reacción de las muestras con ácido fosfórico a $70{ }^{\circ} \mathrm{C}$ mediante un dispositivo GasBench II conectado a un Espectrómetro de Masas Finnigan tipo MAT DeltaXL Plus. El He se utilizó como gas inerte en el sistema siguiendo una metodología adaptada por Spötl y Vennemann (2003). Los resultados fueron calibrados en relación a NBS 19 y reportados en la notación delta convencional con respecto a VPDB o Vienna Pee Dee Belemnitella. La reproducibilidad analítica del método fue de $\pm 0.07 \%$ para $\delta^{13} \mathrm{C}$ y $\pm 0.08 \%$ o para $\delta^{18} \mathrm{O}$, a partir de réplicas de muestras del patrón interno del laboratorio $(\mathrm{n}=6)$.

\subsection{ANÁLISIS ESTADÍSTICO}

El coeficiente de correlación de Pearson se calculó entre $\delta^{13} \mathrm{C}$ y $\delta^{18} \mathrm{O}$, y entre cada isótopo con las variables $\mathrm{PPI}_{550}$ y $\mathrm{PPI}_{1000}$ en las distintas asociaciones de facies de los núcleos AB-1 y AB-2. Las matrices de correlaciones para las diferentes subfacies fueron obtenidas a través de la utilización del paquete Statgraphics (Statgraphics Plus, 2001).

\section{Resultados}

\subsection{COMPOSICIÓN ISOTÓPICA Y ANÁLISIS ESTADÍSTICO DEL NÚGLEO AB-1}

La Tabla 2 muestra las facies identificadas en el núcleo AB-1 por Zanor et al. (2013). La Figura 4 presenta las asociaciones de facies, los valores de PPI550 y PPI1000 (Zanor et al., 2013) y las relaciones isotópicas a lo largo del núcleo AB-1. En la secuencia sedimentaria de AB-1 se identificaron dos asociaciones de facies: AB-1-A (894-792 cm y 694 - $0 \mathrm{~cm}$ ), la cual fue interpretada como una planicie fangosa capilar con sedimentación siliciclástica dominante y AB-1-B $(792-694 \mathrm{~cm})$, representada por un ambiente de laguna perenne con precipitación carbonática, rica en tapices microbianos, que corresponde con un fechamiento ${ }^{14} \mathrm{C}$ de ca. 32506 años cal. AP a los $724 \mathrm{~cm}$ (Tabla 1). 
Tabla 2. Descripción de las facies sedimentarias e interpretación de los ambientes de depósito en los núcleos AB-1 y AB-2. Otras características de las facies se encuentran en Zanor et al. (2013).

\begin{tabular}{|c|c|c|c|c|}
\hline \multicolumn{2}{|c|}{ Facies sedimentarias } & Profundidad (cm) & Indicadores & Ambiente de depósito \\
\hline \multicolumn{5}{|l|}{ Facies 1} \\
\hline Subfacies $1 a$ & Limo masivo & $\begin{array}{l}\text { AB-1: } 894-792 \text { y } 286-0 \\
\text { AB-2: } 176-0\end{array}$ & $\begin{array}{l}\mathrm{PPI}_{550}(2-11 \%) \\
\mathrm{PPI}_{1000}(5-12 \%)\end{array}$ & Planicie fangosa capilar \\
\hline Subfacies $1 b$ & Limo masivo rico en materia orgánica & AB-1: 489-286 & $\begin{array}{l}\mathrm{PPI}_{550}(3-15 \%) \\
\operatorname{PPI}_{1000}(5-9 \%)\end{array}$ & $\begin{array}{l}\text { Planicie fangosa capilar, con lagunas } \\
\text { efímeras ricas en tapices microbianos }\end{array}$ \\
\hline Subfacies $1 c$ & $\begin{array}{l}\text { Limo masivo con láminas carbonáticas } \\
\text { aisladas }\end{array}$ & AB-1: 694-489 & $\begin{array}{l}\mathrm{PPI}_{550}(2-6 \%) \\
\mathrm{PPI}_{1000}(5-10 \%)\end{array}$ & $\begin{array}{l}\text { Planicie fangosa capilar, con lagunas } \\
\text { efímeras }\left(\mathrm{CO}_{3}{ }^{2-}\right)\end{array}$ \\
\hline Facies 2 & Limo laminado a bandeado carbonático & AB-1: 792-694 & $\begin{array}{l}\mathrm{PPI}_{550}(5-12 \%) \\
\mathrm{PPI}_{1000}(7-10 \%)\end{array}$ & Laguna salina perenne $\left(\mathrm{CO}_{3}{ }^{2-}\right)$ \\
\hline Facies 3 & Limo grueso masivo rico en biotita & AB-1: $104.4-94.5$ & $\begin{array}{l}\mathrm{PPI}_{550}(4 \%) \\
\mathrm{PPI}_{1000}(7 \%)\end{array}$ & Corriente mantiforme \\
\hline Facies 4 & Tefra & $\begin{array}{l}\text { AB-2: } 137-121 \\
\text { AB-1: } 859-852 \text { (T4), 111.5-104.5 (T3), } \\
89-84.5 \\
\text { AB-2: } 152-142 \text { (T3), 114.5-104.5 (T2), } \\
16-8 \text { (T1) }\end{array}$ & $\begin{array}{l}\mathrm{PPI}_{550}(4 \%) \\
\mathrm{PPI}_{1000}(7 \%)\end{array}$ & Depósito piroclástico de caída \\
\hline Facies 5 & $\begin{array}{l}\text { Limo masivo, moteado, rico en yeso } \\
\text { intersticial }\end{array}$ & AB-2: 374-176 & $\begin{array}{l}\mathrm{PPI}_{550}(7-15 \%) \\
\mathrm{PPI}_{1000}(8-11 \%)\end{array}$ & Planicie fangosa salina \\
\hline Facies 6 & $\begin{array}{l}\text { Limo laminado a bandeado difuso, } \\
\text { moteado, rico en yeso intersticial. }\end{array}$ & AB-2: 476-374 & $\begin{array}{l}\operatorname{PPI}_{550}(7-15 \%) \\
\operatorname{PPI}_{1000}(8-12 \%)\end{array}$ & $\begin{array}{l}\text { Planicie fangosa salina-Laguna salina } \\
\text { efímera }\left(\mathrm{SO}_{4}{ }^{2-}\right)\end{array}$ \\
\hline Facies 7 & $\begin{array}{l}\text { Láminas limosas-orgánicas-yesíferas } \\
\text { crenuladas }\end{array}$ & AB-2: $538-476$ & $\begin{array}{l}\mathrm{PPI}_{550}(10-18 \%) \\
\operatorname{PPI}_{1000}(6-11 \%)\end{array}$ & $\begin{array}{l}\text { Laguna salina efímera } \mathrm{SO}_{4}{ }^{2-} \text {, } \\
\text { relativamente profunda, dominada por } \\
\text { tapices microbianos }\end{array}$ \\
\hline Facies 8 & Limo masivo con halita & AB-2: $511-5-484.5$ & $\begin{array}{l}\mathrm{PPI}_{550}(8-14 \%) \\
\mathrm{PPI}_{1000}(7-11 \%)\end{array}$ & Laguna salina efímera, $\mathrm{Cl}^{-}$ \\
\hline Facies 9 & Micro-láminas de limo y yeso & AB-2: $576.5-538$ & $\begin{array}{l}\operatorname{PPI}_{550}(12-16 \%) \\
\operatorname{PPI}_{1000}(5-7 \%)\end{array}$ & $\begin{array}{l}\text { Laguna salina efímera, } \mathrm{SO}_{4}{ }^{2-} \text {, altamente } \\
\text { fluctuante }\end{array}$ \\
\hline Facies 10 & Limo masivo a bandeado rico en yeso & AB-2: $612-576.5$ & $\begin{array}{l}\mathrm{PPI}_{550}(13-16 \%) \\
\mathrm{PPI}_{1000}(5-8 \%)\end{array}$ & Laguna salina efímera, $\mathrm{SO}_{4}{ }^{2-}$ \\
\hline Facies 11 & Ritmitas limo-yeso & AB-2: $678-612$ & $\begin{array}{l}\mathrm{PPI}_{550}(11-17 \%) \\
\operatorname{PPI}_{1000}(4-9 \%)\end{array}$ & $\begin{array}{l}\text { Laguna salina efímera, } \mathrm{SO}_{4}{ }^{2-} \text {, somera, } \\
\text { rica en tapices microbianos }\end{array}$ \\
\hline
\end{tabular}

En el núcleo $\mathrm{AB}-1$, la curva de $\delta^{13} \mathrm{C}_{\text {carb }}$ exhibió una variación más acotada que la curva de $\delta^{18} \mathrm{O}_{\text {carb }}$. Las composiciones de $\delta^{13} \mathrm{C}_{\text {carb }}$ alcanzaron un máximo de $-9.11 \%$ o (100.5 cm) y un mínimo de $-3.44 \%$ $(187 \mathrm{~cm}$; promedio $=-5.13 \%$ ). Por su parte, los valores de $\delta^{18} \mathrm{O}_{\text {carb }}$ fluctuaron entre -16.19 (490 cm) y $2.06 \%$ (195 cm; promedio $=-3.93 \%$, evidenciando entre ambos datos una diferencia de 18 unidades. El registro isotópico de AB-1 en su conjunto no presentó correlación significativa entre las variables analizadas.

\subsubsection{ASOCIACIÓN DE FACIES AB-1-A (894- 792 Y $694-$ $0 \mathrm{~cm})$}

En términos generales, AB-1-A exhibió composiciones negativas de $\delta^{13} \mathrm{C}_{\text {carb }}$, con variaciones entre -9.11 y $-3.44 \%$ (promedio $=-5.10 \%$ ). Los valores de $\delta^{18} \mathrm{O}_{\text {carb }}$ fluctuaron desde -16.19 hasta 2.06
$\%$ o promedio $=-3.93 \%$; Figura 4$).$ El grado de asociación entre las variables analizadas fue nulo al no resultar ningún coeficiente de correlación significativo.

En la Subfacies 1c (limo masivo con láminas carbonáticas aisladas: $694-489 \mathrm{~cm})$ se registró el valor más negativo de $\delta^{18} \mathrm{O}_{\text {carb }}$ de toda la secuencia AB-1, alcanzando los $-16.19 \%$ o (490 cm de profundidad). La subfacies $1 \mathrm{~b}(489-286 \mathrm{~cm}$ : limo masivo rico en materia orgánica) alcanzó un promedio de $-4.79 \%$ para $\delta^{13} \mathrm{C}_{\text {carb }}$ y $-3.60 \%$ para $\delta^{18} \mathrm{O}_{\text {carb }}$. En los limos masivos de la Subfacies la $(894-792 \mathrm{~cm}$ y $286-0 \mathrm{~cm})$ se encontró el valor más positivo de $\delta^{18} \mathrm{O}_{\text {carb }}$ de todo el núcleo $\mathrm{AB} 1$ $(2.06$ \%o: $195 \mathrm{~cm})$. Una correlación positiva significativa se registró entre $\mathrm{PPI}_{550}$ y $\mathrm{PPI}_{1000}(\mathrm{r}=0.72, \mathrm{p}$ $=0.0016^{* *}, \mathrm{n}=16$ ), así como entre $\mathrm{PPI}_{1000}$ y $\delta^{13} \mathrm{C}$ carb $\left(r=0.55, p=0.0259^{*}, \mathrm{n}=16\right)$. Finalmente, $\mathrm{a}$ 


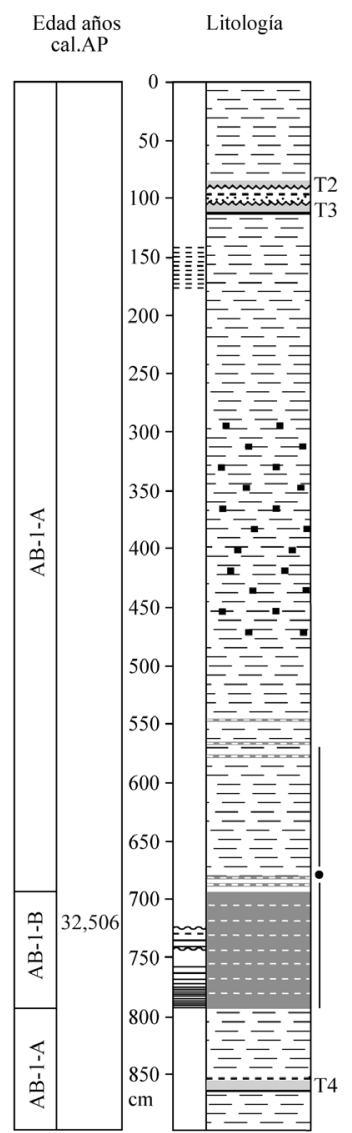

Facies sedimentarias
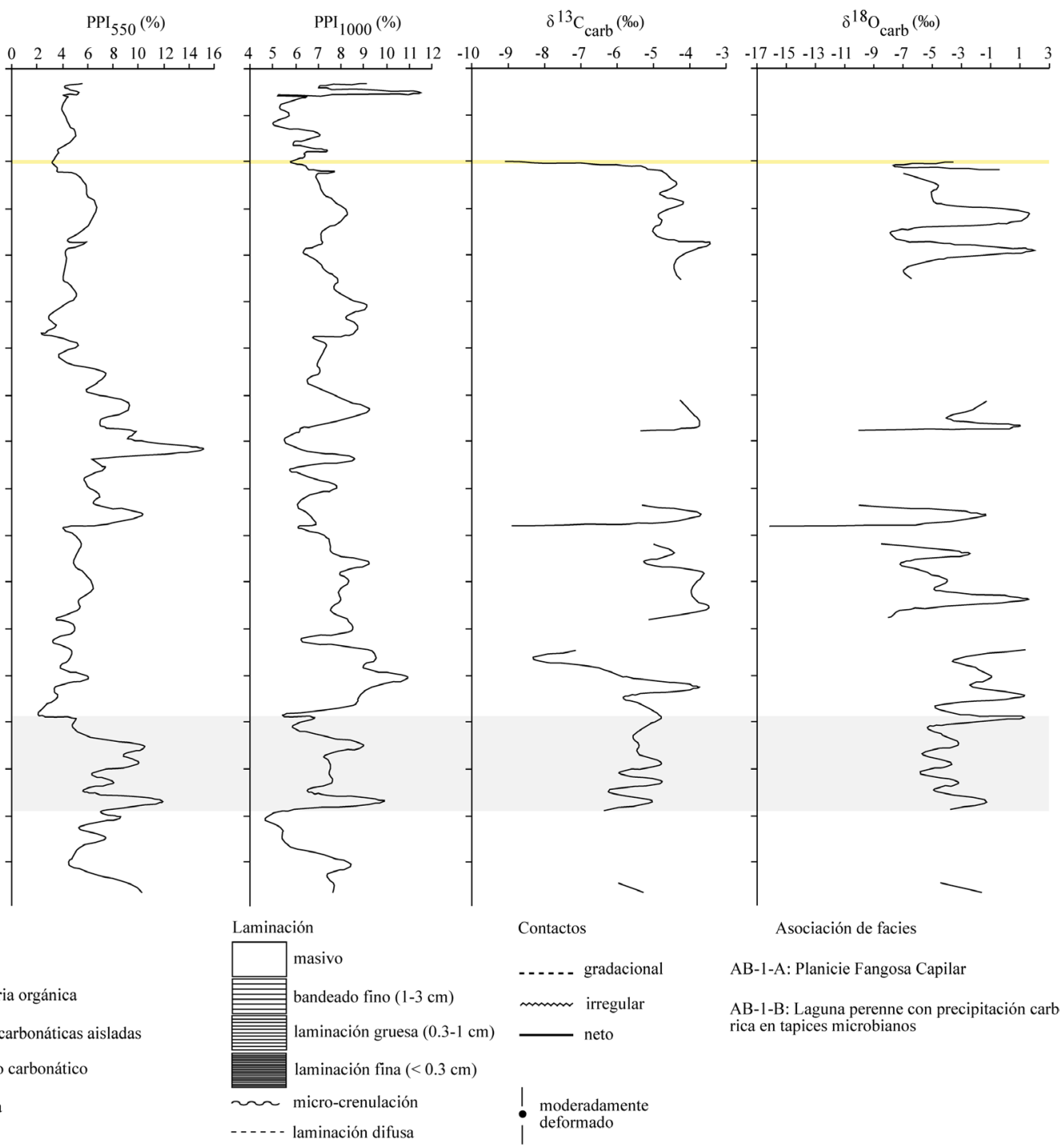

$=7$ la Limo masivo

E $1 \mathrm{~b}$ Limo masivo rico en materia orgánica

$=$

- lc Limo masivo con láminas carbonáticas aisladas

2 Limo laminado a bandeado carbonático

$\because \because \vdots 3$ Limo grueso rico en biotita

T 4 Tefra

-..-- laminación difusa

deformado

Figura 4 Valores de $\mathrm{PPI}_{550}, \mathrm{PPI}_{1000}(\%), \delta^{13} \mathrm{C}_{\text {carb }}$ y $\delta^{18} \mathrm{O}_{\text {carb }}(\%)$ en el núcleo $\mathrm{AB}-1$. En colores blancos y grises se muestran las distintas asociaciones de facies. En color amarillo se indica el depósito de limo grueso.

los $100.5 \mathrm{~cm}$ de profundidad se exhibió la relación isotópica del $\mathrm{C}$ más negativa de AB-1 (-9.11\%), representada por un nivel de limo grueso masivo rico en biotita de la Facies 3 .

\subsubsection{ASOCIACIÓN DE FACIES AB-1-B $(792-694 \mathrm{~cm})$}

En la Facies 2 (limo laminado a bandeado carbonático: $792-694 \mathrm{~cm}$; Tabla 2), tanto los valores de $\delta^{13} \mathrm{G}_{\text {carb }}$ como los de $\delta^{18} \mathrm{O}_{\text {carb }}$, exhibieron fluctuaciones menores en comparación con las otras facies sedimentarias del núcleo AB-1 (Figura 4).
Las composiciones $\delta^{13} \mathrm{C}_{\text {carb }}$ variaron desde -4.73 a $-6.25 \%$ (promedio: $-5.30 \%$ ) mientras que las concentraciones de $\delta^{18} \mathrm{O}_{\text {carb }}$ oscilaron entre -5.90 y $1.36 \%$ (promedio: $-3.59 \%$ ). Dentro de AB1-B se obtuvo una correlación positiva altamente significativa entre $\mathrm{PPI}_{550}$ y $\mathrm{PPI}_{1000}(\mathrm{r}=0.88, \mathrm{p}=$ $0.0008 * *, \mathrm{n}=10)$. Esta es la única sección en la que se presentó una correlación positiva entre los isótopos $\delta^{13} \mathrm{C}_{\text {carb }}$ y $\delta^{18} \mathrm{O}_{\text {carb }}(\mathrm{r}=0.62, \mathrm{p}=0.0541$, $\mathrm{n}=10)$. 


\subsection{GOMPOSIGIONES ISOTÓPICAS Y ANÁLISIS ESTADÍSTICO DEL NÚGLEO AB-2}

Las facies sedimentarias en el núcleo AB-2 y las interpretaciones de sus ambientes de depósito (Zanor et al., 2013) son mostradas en la Tabla 2. La Figura 5 muestra las asociaciones de facies, los valores de PPI $_{550}$ y PPI ${ }_{1000}$ (Zanor et al., 2013), y las composiciones isotópicas a lo largo del núcleo AB-2. Las 5 asociaciones de facies reconocidas en la columna sedimentaria de AB-2 fueron las siguientes: 1) AB-2-A (678 - $538 \mathrm{~cm})$ : ambiente de laguna efímera somera con precipitación sulfatada dominante que correlaciona con edades de radio- carbono de $c a .33514$ y 30579 años cal. AP (638 y $612 \mathrm{~cm}$, respectivamente; Tabla 1); 2) AB-2-B (538 $-476 \mathrm{~cm})$ : ambiente de laguna salina relativamente profunda con precipitación sulfatada, dominada por tapices microbianos que corresponde con una edad de radiocarbono de ca. 25607 años cal. AP, a los $514 \mathrm{~cm}$; Tabla 1); 3) AB-2-C (476-374 cm): ambiente de laguna efímera que alterna con una planicie fangosa salina; 4) AB-2-D $(374-176 \mathrm{~cm})$ : ambiente de planicie fangosa salina, y 5) AB-2-E $(176-0 \mathrm{~cm})$ : ambiente de planicie fangosa capilar. El núcleo AB-2 presentó relaciones isotópicas contrastantes en cada una de las asociaciones de facies identificadas (Figura 5). A lo largo de toda

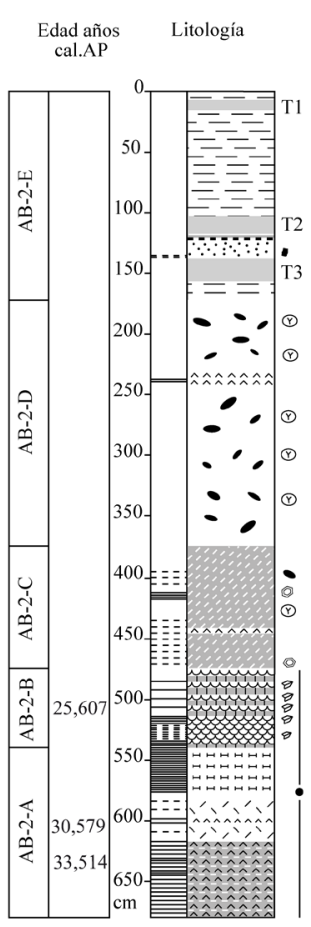

Facies sedimentarias

E 1 Limo masivo

$\because 3$ Limo grueso rico en biotita

T 4 Tefra

-1 5 Limo masivo, moteado, rico en yeso intersticial

283. 6 Limo laminado a bandeado difuso,

moteado, rico en yeso intersticial

7 Láminas limosas-orgánicas-yesíferas

crenuladas

||||| 8 Limo masivo con halita

본변 9 Micro-láminas de limo y yeso

10 Limo masivo a bandeado rico en yeso

춧ㅊㅅㅡ 11 Ritmitas limo-yeso

Figura 5 Valores de $\mathrm{PPI}_{550}, \mathrm{PPI}_{1000}(\%), \delta^{13} \mathrm{C}_{\text {carb }}$ y $\delta^{18} \mathrm{O}_{\text {carb }}(\%$ ) en el núcleo $\mathrm{AB}-2$. Las distintas tonalidades de grises a blanco muestran las diferentes asociaciones de facies. En color amarillo se indica el nivel de limo grueso.

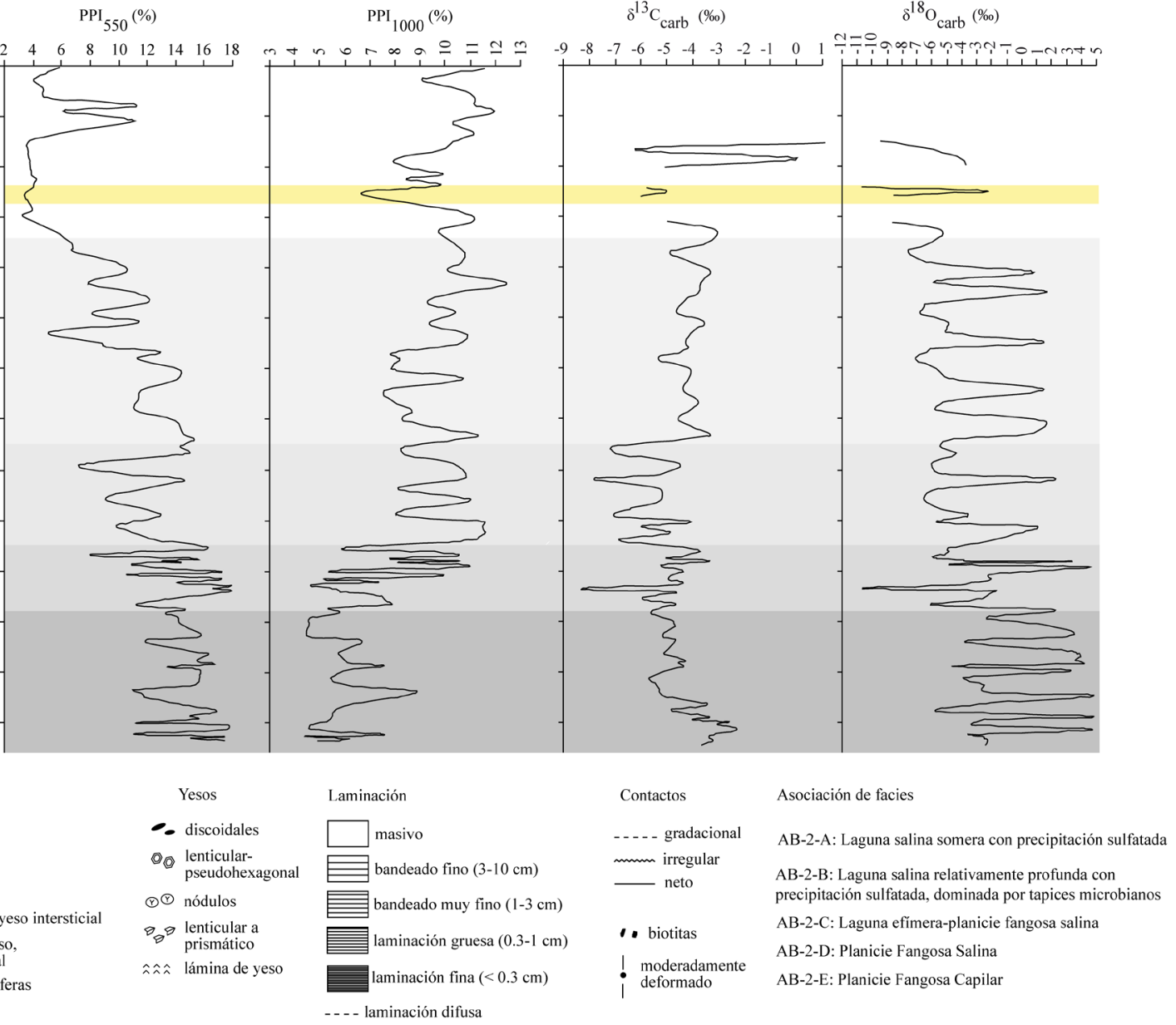


la secuencia sedimentaria, el $\delta^{13} \mathrm{C}_{\text {carb }}$ se mantuvo en rangos de valores más estrechos dentro de cada asociación de facies en comparación con el $\delta^{18} \mathrm{O}$ carb. En el intervalo comprendido desde la base del testigo $(678 \mathrm{~cm})$ hasta los $377 \mathrm{~cm}(\mathrm{AB}-2-\mathrm{A}, \mathrm{AB}-$ 2-B, AB-2-C; Figura 5), los valores de $\delta^{13} \mathrm{C}_{\text {carb }}$ exhibieron una tendencia decreciente y fueron los más negativos registrados mientras que a partir de esa profundidad hasta los $129 \mathrm{~cm}$ (AB-2-D), las composiciones de $\delta^{13} \mathrm{C}_{\text {carb }}$ se volvieron más positivas, manteniéndose relativamente constantes. La secuencia entre 129 y 75 cm (AB-2-E) se caracterizó por una gran variación en los valores de $\delta^{13} \mathrm{C}_{\text {carb }}$, encontrándose entre -8.29 y $0.90 \%$ (promedio = $-4.56 \%$ ).

La curva del $\delta^{18} \mathrm{O}_{\text {carb }}$ mostró una gran variabilidad en todo el núcleo AB-2, con una distribución de valores heterogénea, entre - 10.69 y 4.86 \%o (promedio $=-2.85 \%$; diferencia de 14 unidades; Figuras 5 y 6). La curva mostró una tendencia decreciente desde los niveles profundos a los superficiales. Con base en los valores de $\delta^{18} \mathrm{O}_{\text {carb }}$ se distinguieron 6 poblaciones principales de datos, desde los más negativos a los más positivos (Grupos 1 a 6; Figura 6), característicos de cada asociación de facies de AB-2: Grupo 1 (-11.00 a -10.00 \%o); Grupo 2 (-9.00 a -8.00\%); Grupo 3 (-8.00 a $-4.00 \%)$; Grupo 4 (-4,00 a -1.50 \%o); Grupo 5 (0.00 a 2.50 \%), y Grupo 6 (3.00 a $5.00 \%$ \%). La mayoría de los valores de $\delta^{18} \mathrm{O}_{\text {carb }}$ en $\mathrm{AB}-2$ se encontraron dentro de los Grupos 3 y 4 (-8.00 a -1.50; Figura 6).

A diferencia del patrón isotópico de AB-1, el núcleo AB-2 exhibió una correlación negativa y altamente significativa entre $\mathrm{PPI}_{550}$ y $\mathrm{PPI}_{1000}(\mathrm{r}=-0.60$, $\mathrm{p}=0.0000^{* *}, \mathrm{n}=89$ ).

\subsubsection{ASOCIACIÓN DE FACIES AB-2-A $(678-538 \mathrm{~cm})$}

En general, el depósito de AB-2-A se caracterizó por presentar valores de $\delta^{13} \mathrm{C}_{\text {carb }}$ que alcanzaron un promedio de $-4.32 \%$ o (-5.68 a $-2.24 \%$ y y las composiciones promedios más enriquecidas de $\delta^{18} \mathrm{O}$ carb de todo el núcleo AB-2, con un valor de -0.44 \%o (-5.58 a +4.88\%o; Figura 6). El PPI 550 aumentó mientras que el PPI ${ }_{1000}$ disminuyó, mostrando una correlación negativa y altamente significativa $(\mathrm{r}=$ $-0.79, \mathrm{p}=0.0000 * *, \mathrm{n}=26$ ).

En la Facies 11 (Ritmitas limo-yeso: $678-612 \mathrm{~cm}$ ), los valores de $\delta^{13} \mathrm{C}_{\text {carb }}$ fluctuaron entre -5.41 a -2.24 $\%$ o (promedio $=-3.87 \%$ ) mientras que la mayoría de las relaciones ${ }^{18} \mathrm{O} /{ }^{16} \mathrm{O}$ en los carbonatos pertenecieron al Grupo 4 (Figura 6). En esta facies, se encontró una correlación positiva significativa entre $\delta^{13} \mathrm{C}_{\text {carb }}$ y $\mathrm{PPI}_{550}(\mathrm{r}=0.5446, \mathrm{p}=0.0441$ *, $\mathrm{n}=14$ ), correlaciones negativas $\mathrm{y}$ altamente significativas entre $\delta^{13} \mathrm{C}_{\text {carb }}$ y $\mathrm{PPI}_{1000}(\mathrm{r}=-0.68, \mathrm{p}$ $=0.0071^{* *}, \mathrm{n}=14$ ) y finalmente, entre $\mathrm{PPI}_{550} \mathrm{y}$ $\mathrm{PPI}_{1000}\left(\mathrm{r}=-0.96, \mathrm{p}=0.0000^{* *}, \mathrm{n}=14\right)$. La Facies 10 o de Limo masivo a bandeado rico en yeso $(612$ $-576.5 \mathrm{~cm}$ ) alcanzó un valor promedio de $\delta^{18} \mathrm{O}$ carb de $-4.84 \%$ para $\delta^{13} \mathrm{C}_{\text {carb }}$ y en general, valores positivos correspondientes al Grupo 6 (Figura 6). Los valores de $\delta^{13} \mathrm{C}_{\text {carb }}$ de la Facies 9 (Micro-láminas de limo y yeso: $576.5-538 \mathrm{~cm}$ ) mostraron un promedio de $-5.04 \%$ y para las composiciones de $\delta^{18} \mathrm{O}_{\text {carb }}$ fueron dominantes los valores del Grupo 4 y 6 (Figura 6). En este caso, el grado de asociación entre PPI $_{550}$ y PPI $_{1000}$ se estableció mediante una correlación negativa significativa $(\mathrm{r}=-0.92, \mathrm{p}=$ $0.0279 *, n=5)$.

\subsubsection{ASOCIACIÓN DE FACIES AB-2-B $(537-476 \mathrm{~cm})$}

AB-2-B obtuvo una composición promedio para $\delta^{13} \mathrm{G}_{\text {carb }}$ que alcanzó los $-4.76 \%$ y valores más negativos de $\delta^{18} \mathrm{O}_{\text {carb }}$ en comparación con la asociación de facies AB-2-A (promedio $=-2.94 \%$; Figuras 5 y 6). A medida que PPI ${ }_{550}$ aumentó, PPI $_{1000}$ disminuyó $\left(\mathrm{r}=-0.84, \mathrm{p}=0.0000^{* *}, \mathrm{n}=20\right.$ ).

En particular, los sedimentos de la Facies 8 (Limo masivo con halita: $511.5-484.5 \mathrm{~cm}$ ) presentaron composiciones promedio de $\delta^{13} \mathrm{C}_{\text {carb }}$ de $-4.43 \%$ y un predominio de valores de $\delta^{18} \mathrm{O}_{\text {carb }}$ pertenecientes a los Grupos 3, 4 y 6. Por su parte, el registro de la Facies 7 o Láminas limosas-orgánicas-yesíferas crenuladas $(538-476 \mathrm{~cm})$ registró composiciones de $\delta^{13} \mathrm{C}_{\text {carb }}$ muy negativas (promedio $=-5.55 \%$ ) y valores de $\delta^{18} \mathrm{O}_{\text {carb }}$ pertenecientes principalmente a los Grupos 3 y 4 (Figura 6), y en menor medida, 


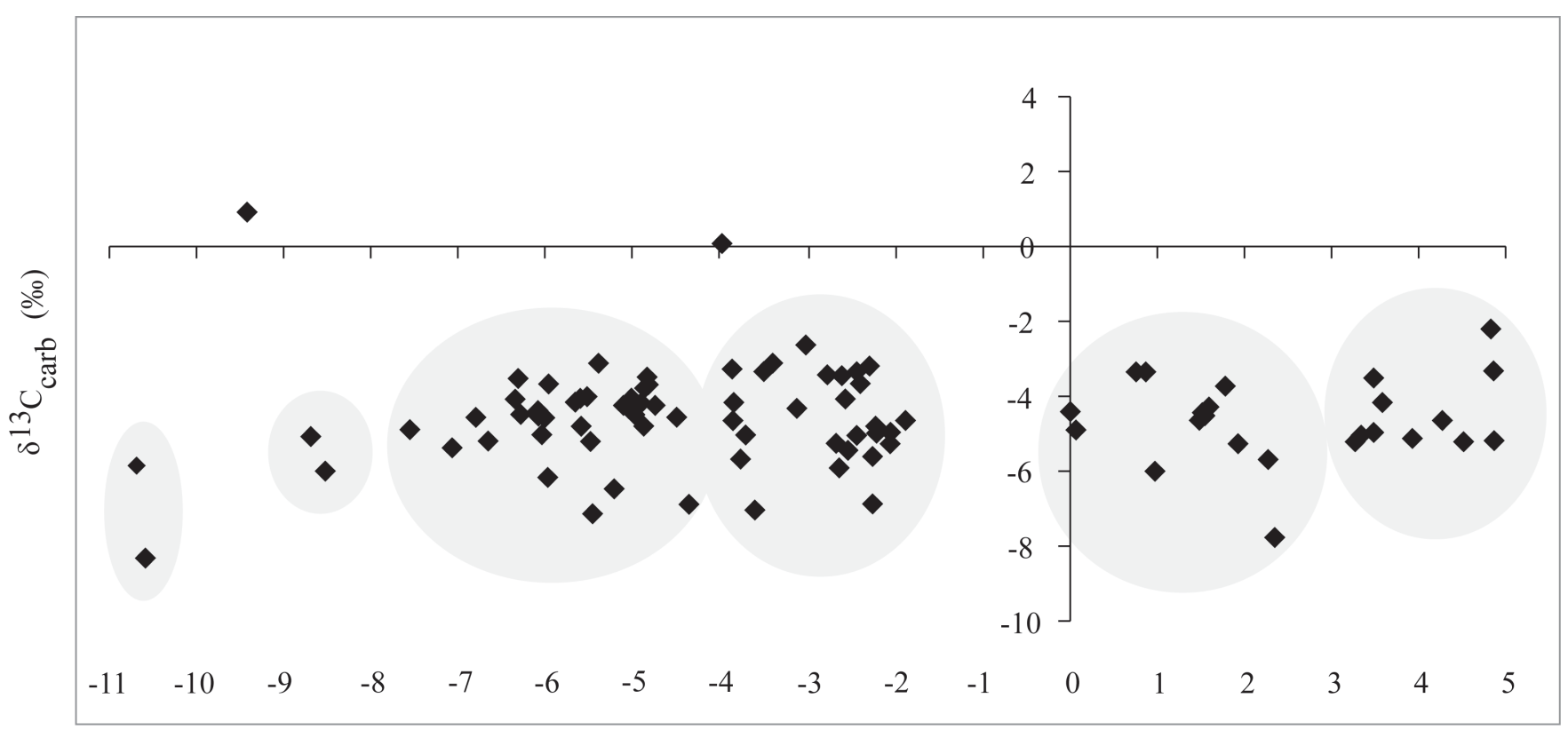

$$
\delta^{18} \mathrm{O}_{\text {carb }}(\%)
$$

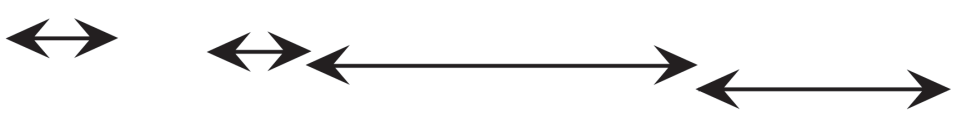

Grupo 1 Grupo 2 Grupo 3

AB-2-B

AB-2-E

AB-2-E
AB-2-A

AB-2-B

AB-2-C

AB-2-D

AB-2-E
Grupo 4

$\mathrm{AB}-2-\mathrm{A}$

AB-2-B

AB-2-C

AB-2-E

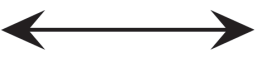

Grupo 5

AB-2-B

AB-2-C

AB-2-D

AB-2-E

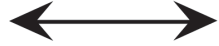

Grupo 6

$\mathrm{AB}-2-\mathrm{A}$

AB-2-B

Figura 6 Grupos $(1-6)$ formados por los valores de $\delta^{18} \mathrm{O}_{\text {carb }}$ en el núcleo $A B-2$.

de los Grupos 1 (mínimo = -10.59\%o) y 5 (máximo $=1.94 \% 0$ ).

\subsubsection{ASOCIACIÓN DE FACIES AB-2-C $(476-374 \mathrm{~cm})$}

La Facies 6 (Limo laminado a bandeado difuso, moteado, rico en yeso intersticial: $476-374 \mathrm{~cm}$ ) registró los valores promedio de $\delta^{13} \mathrm{C}_{\text {carb }}$ más negativos de todo el relleno sedimentario (promedio $=$ $-5.93 \%$; Figura 5). Entre los valores del isótopo de $\mathrm{O}$ dominantes se encontraron mayormente los representados por los Grupos 3 y 4 (Figura 7), con excursiones positivas del Grupo 5. En particular, en esta facies se exhibió una correlación inversa altamente significativa entre $\mathrm{PPI}_{550}$ y $\delta^{13} \mathrm{C}_{\text {carb }}(\mathrm{r}=$ $\left.-0.79, \mathrm{p}=0.0013^{* *}, \mathrm{n}=13\right)$.

\subsubsection{ASOCIACIÓN DE FACIES AB-2-D $(374-176 \mathrm{~cm})$}

Los valores de $\delta^{13} \mathrm{G}_{\text {carb }}$ en la facies 5 (Limo masivo, moteado, rico en yeso intersticial: $374-176 \mathrm{~cm}$ ) alcanzaron un promedio de $-4.14 \%$ mientras que las relaciones isotópicas del $\mathrm{O}$ en los carbonatos se encontraron en su mayor parte dentro del Grupo 3, con picos positivos correspondientes al Grupo 5 (Figura 6). 


\subsubsection{ASOCIACIÓN DE FACIES AB-2-E $(176-0 \mathrm{~cm})$}

En esta asociación de facies los valores de $\delta^{13} \mathrm{C}$ carb fluctuaron en un amplio rango, alcanzando un promedio de -3.91 \%o (Figura 5). AB-2-E presentó los dos únicos valores positivos de $\delta^{13} \mathrm{C}_{\text {carb }}$ de todo el núcleo $(0.90 \%$ a los $75 \mathrm{~cm}$ y $0.07 \%$ a los 92 $\mathrm{cm})$. AB-2-E se caracterizó por presentar valores de $\delta^{18} \mathrm{O}_{\text {carb }}$ dentro de las poblaciones más negativas (Grupos 1 a 4; promedio: -6.52; Figura 6), estando ausentes las composiciones más enriquecidas de los Grupos 5 y 6.

Los intervalos limosos masivos de la Facies 1 (176 $-0 \mathrm{~cm})$ mostraron composiciones de $\delta^{13} \mathrm{C}_{\text {carb }}$ con un valor promedio de $-3.65 \%$ mientras que las relaciones ${ }^{18} \mathrm{O} /{ }^{16} \mathrm{O}$ en los carbonatos registraron un promedio de $-6.12 \%$. Los sedimentos de la Facies 3 (Limo grueso masivo rico en biotita: 137 - $121 \mathrm{~cm}$ ) presentaron composiciones de $\delta^{13} \mathrm{C}_{\text {carb }}$ de -5.59 y se caracterizaron por valores de $\delta^{18} \mathrm{O}_{\text {carb }}$ muy negativos (Grupos 1, 2 y 4; Figura 6), con un valor promedio de $-7.14 \%$ (mínimo $=-10.69 \%$ a los $121 \mathrm{~cm}$ de profundidad).

\section{Discusión}

A partir del patrón isotópico analizado a lo largo del relleno sedimentario de la Salina de Ambargasta y su correlación con otros indicadores ambientales $\left(\mathrm{PPI}_{550}\right.$ y $\left.\mathrm{PPI}_{1000}\right)$ junto al análisis de facies y el modelo cronológico obtenido (desde el Pleistoceno tardío; Zanor et al., 2013; Tabla 1 y Figura 3), se plantea la reconstrucción paleohidrológica de un sistema playa ubicado en la zona oriental de la DA del continente sudamericano (Figura 7).

Con base en lo propuesto por Talbot (1990), el grado de asociación prácticamente nulo entre $\delta^{13} \mathrm{G}_{\text {carb }}$ y $\delta^{18} \mathrm{O}_{\text {carb }}$ en ambas secuencias sedimentarias (AB-1 y AB-2) evidenció que el sistema no se habría comportado como hidrológicamente cerrado durante un lapso continuo de aproximadamente 30000 años aunque si existió un intervalo de tiempo en el que se registró encerramiento hidrológico (39600 - 26700 años cal. AP: Asociación de facies AB-1-B; Figura 7). La ausencia de relación entre $\delta^{13} \mathrm{C}_{\text {carb }}$ y $\delta^{18} \mathrm{O}_{\text {carb }}$ en ambos rellenos indicó que el sistema probablemente sufrió variaciones en las entradas/salidas de agua subterránea o que los isótopos no respondieron con la misma intensidad ni de manera sincrónica a los factores forzantes internos y externos del microambiente (principalmente aportes de agua dulce y cambios en la evaporación; Li y Ku, 1997).

La reconstrucción comienza con un periodo comparativamente más seco ocurrido aproximadamente entre los ca. 44700 y 39600 años cal. AP, representado por una planicie fangosa capilar con sedimentación siliciclástica dominante (Figura 7). Zárate y Tripaldi (2012) reconocieron depósitos arenosos y loéssicos cubriendo gran parte de las Pampas Argentinas asociados a un evento eólico de importancia ocurrido durante el Pleistoceno tardío (42700 y 30000 años cal. AP). Siguiendo el criterio de Zárate (2003), el nivel de tefra de origen distal intercalado con los limos masivos en AB-1 (Facies 4: 859 - $852 \mathrm{~cm}$ ) avalaría un incremento en el transporte y sedimentación por el viento bajo condiciones más áridas durante esta etapa.

Posteriormente una fase más húmeda y cálida se registró a los ca. 39600 - 26700 años cal. AP (Figura 7). Este aumento en la humedad efectiva sería correlacionable con el MIS 3 (Wright, 2000), definido en la región sur de Sudamérica a los ca. 60000 - 50000 a 28000 años cal. AP (Rabassa y Ponce, 2013). Un episodio cálido y húmedo fue reconocido a los ca. 30000 años AP en la región Pampeana Argentina, representado por una comunidad de mamíferos adaptados a estas condiciones climáticas (Prado y Alberdi, 1999). Un subambiente de laguna efímera somera con precipitación sulfatada (AB-2-A: $678-538 \mathrm{~cm})$ se instaló en la zona central de Ambargasta mientras que en el borde este se desarrolló una laguna perenne con precipitación carbonática, rica en tapices microbianos (AB-1-B: 792 - 694 cm; Figura 7). Los valores promedios negativos de $\delta^{13} \mathrm{C}_{\text {carb }}$ y $\delta^{18} \mathrm{O}_{\text {carb }}$ avalaron la expansión de lagunas alimentadas por agua más dulce (agua subterránea, escorrentías y precipitación) durante una etapa con balance hídrico positivo dominante. En AB-2-A, las composiciones 


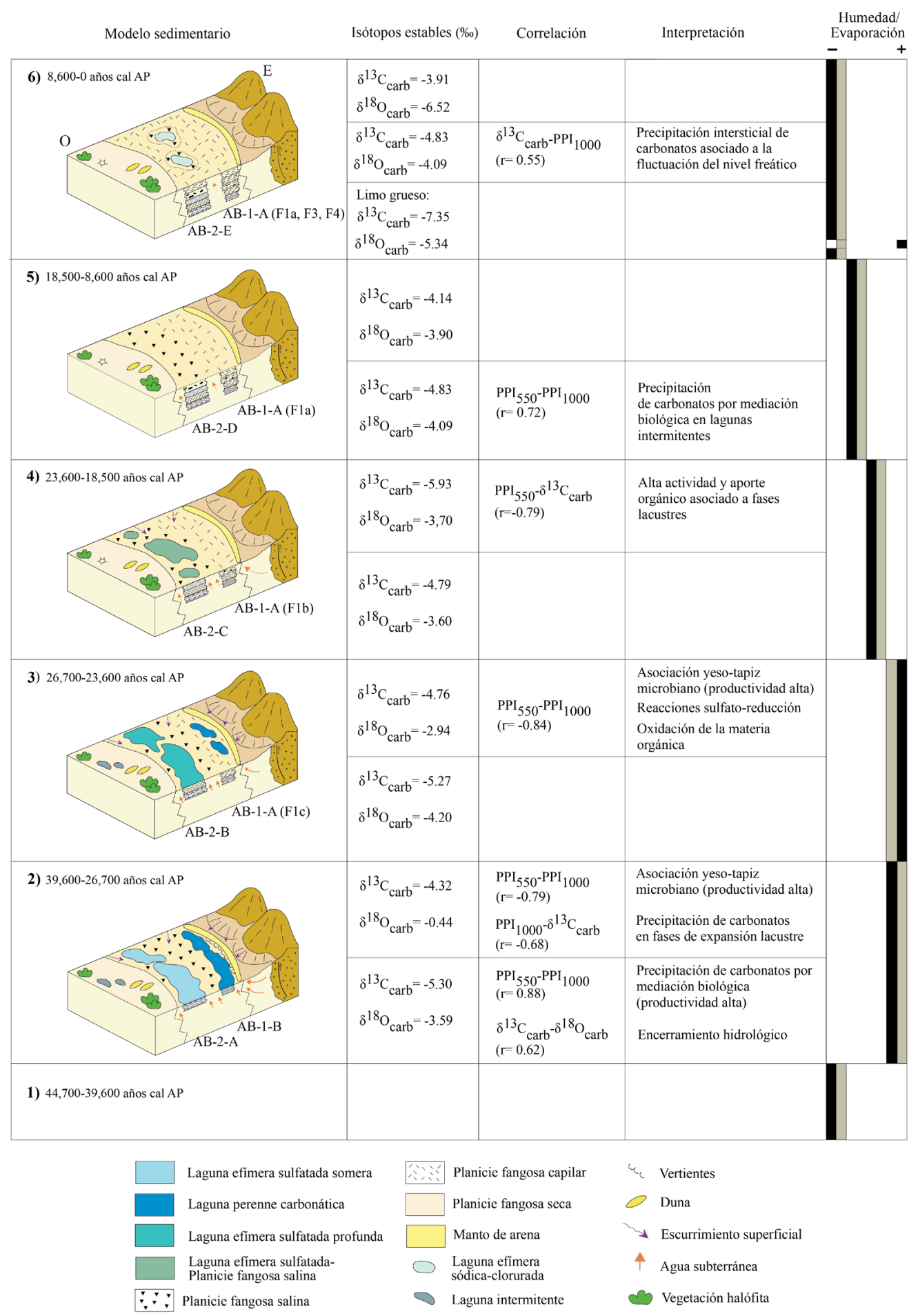

Figura 7 Modelo de sedimentación y evolución hidroclimática a lo largo del Pleistoceno tardío y Holoceno en la Salina de Ambargasta. Los valores promedio de $\delta^{13} \mathrm{C}_{\text {carb }}$ y $\delta^{18} \mathrm{O}_{\text {carb }}$ (\%) y las correlaciones de Pearson se muestran para cada asociación de facies de los núcleos $A B-1$ y AB-2, junto a sus interpretaciones paleoambientales. Las flechas indican el aporte de agua subterránea. En la columna "Isótopos estables" las concentraciones se indican en valores promedio, mostrando en la parte superior los valores para $A B-2$ y en la parte inferior para AB-1. Las barras negras representan la humedad y las barras grises la evaporación. Las asociaciones de facies se muestran en las Figuras 4 y 5. 
de $\delta^{18} \mathrm{O}_{\text {carb }}$ alternantes entre negativas y positivas indicarían una alta sensibilidad de las lagunas a las fluctuaciones en los aportes acuosos y a la tasa de evaporación. Según el patrón isotópico resultante y las correlaciones de Pearson entre los isótopos estables y entre cada isótopo con los contenidos de PPI $_{550}$ y PPI ${ }_{1000}$ existieron diferencias amplias entre la dinámica de sedimentación de AB-2-A y $\mathrm{AB}-1-\mathrm{B}$. Las variaciones más estrechas en las relaciones de los isótopos de $\mathrm{C}$ y $\mathrm{O}$ en el registro de $\mathrm{AB}-1-\mathrm{B}\left(\delta^{13} \mathrm{C}_{\text {carb }}:-2 \%\right.$ y $\delta^{18} \mathrm{O}_{\text {carb }}: \sim-4 \%$ sugirieron una similitud entre la composición isotópica de los aportes acuosos y la laguna. Asimismo, un flujo de agua continuo permitió el mantenimiento del ambiente acuático por un periodo prolongado de tiempo sin alcanzar una fase de desecación total. El aporte de vertientes en las zonas marginales de Ambargasta contribuiría a aumentar la dilución de este ambiente lacustre, ingresando agua rica en ${ }^{12} \mathrm{C}$ y ${ }^{16} \mathrm{O}$. Como fuera señalado por Zanor et al. (2012), en el borde oriental del ambiente evaporítico moderno existen depósitos de vertientes bien desarrollados (duricostras de calcretes-silcretes) que indican la importancia de la contribución subterránea en la alimentación y sedimentación de Ambargasta. Dargám (1995) efectuó un estudio de la hidroquímica en los ambientes sedimentarios de las Salinas Grandes (ambiente evaporítico aledaño a Ambargasta; Figura 1), donde registraron $\mathrm{pH}$ cercanos a 9.0 en las vertientes localizadas en los borde de la salina, argumentando el aumento de alcalinidad por la acrecencia de la actividad fotosintética y por pérdida de $\mathrm{CO}_{2}$ hacia la atmósfera, al emerger los acuíferos subterráneos. En AB-1-B la correlación positiva altamente significativa entre PPI $_{550}$ y PPI $_{1000}(r=0.88)$ reafirmó una mediación biológica en la precipitación carbonática de la laguna perenne a través de la participación de tapices microbianos, reflejando un periodo de mayor productividad orgánica. Según Visscher y Stolz (2005), la precipitación neta de los carbonatos en ambientes salinos es inducida por procesos de fotosíntesis a través de la actividad metabólica de microorganismos en conjunto con reacciones geoquímicas (abióticas; por ej., evaporación). Los picos positivos en las composiciones isotópicas del $\mathrm{C}$ en los carbonatos podrían explicarse por un aumento en la fotosíntesis acuática, enriqueciendo el reservorio del CIDT en ${ }^{13} \mathrm{C}$ (McKenzie y Hollander, 1993; Valero-Garcés et al., 2000). Por otra parte, la curva del $\delta^{18} \mathrm{O}_{\text {carb }}$ en AB-2-A mostró una distribución muy heterogénea de valores (Grupos 3 a 6: -5.58 a $+4.88 \%$; Figura 7) lo que indicaría grandes variaciones en los mecanismos principales de fraccionamiento isotópico. La homogeneidad tipológica y de tamaños de los cristales de yeso precipitados en las lagunas efimeras durante este periodo junto a las laminaciones fangosas-yesíferas de espesores delgados permitió interpretar para AB-2-A fases alternantes de expansión lacustre seguidas de desecación con precipitación evaporítica (Zanor et al., 2013), lo cual está reflejado por la alta variabilidad en las relaciones isotópicas (Figura 5). Con base en Lamb et al. (2002), relaciones P/E bajas conducen a un aumento de $\delta^{18} \mathrm{O}$ debido a un incremento en la evaporación mientras que el $\delta^{13} \mathrm{C}_{\text {carb }}$ se incrementa por la pérdida de $\mathrm{CO}_{2}$ desde el agua hacia la atmósfera, el intercambio gaseoso con el $\mathrm{CO}_{2}$ atmosférico o por una intensificación de la actividad fotosintética por parte de microorganismos. Por otro lado, cuando la relación $\mathrm{P} / \mathrm{E}$ es alta, los ambientes lacustres experimentan dilución por el aporte de aguas isotópicamente más livianas resultando valores de $\delta^{13} \mathrm{C}_{\text {carb }}$ y $\delta^{18} \mathrm{O}_{\text {carb }}$ empobrecidos. De manera similar, Lamb et al. (2000) reportaron un rango de fluctuación amplio entre los valores máximos y mínimos de $\delta^{18} \mathrm{O}_{\text {carb }}(-8.00 \mathrm{a}+4.00 \%$ o) en el lago Tilo (Etiopía), adjudicando parte de esta señal a grandes cambios en la contribución subterránea. En esta secuencia sedimentaria, la correlación negativa altamente significativa entre $\mathrm{PPI}_{550}$ y $\mathrm{PPI}_{1000}(\mathrm{r}=-0.79)$ indicaría una productividad primaria en coincidencia con los niveles donde domina la precipitación de sulfatos. Canfield et al. (2004) reportaron la colonización de comunidades microbianas en costras yesíferas de ambientes hipersalinos, documentando una amplia adaptación y actividad metabólica de las colonias de bacterias dentro de las evaporitas ("tapices microbia- 
nos endoevaporíticos"; Rothschild et al., 1994). De la misma forma, Zanor et al. (2013) describió en el registro sedimentario de AB-2 asociaciones fangosas-orgánicas-yesíferas desarrolladas en las salmueras efimeras a lo largo de un ciclo depositacional (etapas de inundación, concentración evaporítica y desecación). La correlación negativa altamente significativa entre PPI ${ }_{1000}$ y $\delta^{13} \mathrm{G}_{\text {carb }}(\mathrm{r}=$ -0.68: Facies 11; Figura 7) avalaría que la precipitación de carbonatos en las lagunas sulfatadas es dominante en las etapas de expansión lacustre mientras que la cristalización de sulfatos ocurre en las etapas de concentración evaporítica. El fraccionamiento químico y la modificación en la especiación del CIDT se producirían por aumentos en la evaporación y un mayor intercambio gaseoso de $\mathrm{CO}_{2}$ hacia la atmósfera, representado por picos positivos de $\delta^{13} \mathrm{C}_{\text {carb }}$. Adicionalmente, los procesos de degradación de la materia orgánica en el fondo de las lagunas sulfatadas pudieron haber influido limitando la presencia de carbonatos en estos sedimentos. Con base en lo propuesto por Visscher y Stolz (2005), la oxidación de la materia orgánica a través de la respiración de bacterias aerobias heterótrofas produce una disminución del $\mathrm{O}_{2}$ y una consecuente reducción del $\mathrm{pH}$ del medio, lo que conllevaría a la disolución de los carbonatos lacustres. Un estudio realizado por Dargám y Depetris (1995) en las Salinas Grandes de Córdoba (Argentina; Figura 1) demostraron que en las lagunas efimeras de composición principalmente sulfatada las concentraciones de carbonatos muy bajas le confieren a las aguas una mínima capacidad reguladora, por lo que cualquier actividad biológica de consumo o producción de $\mathrm{CO}_{2}$ produciría una marcada variación en el $\mathrm{pH}$, alcalinidad, CIDT u oxígeno disuelto, promoviendo la precipitación o disolución de los carbonatos.

Una fase hídrica más positiva que la anterior se evidenció a los ca. 26700 - 23600 años cal. AP (Figura 7). Este estadio evolutivo húmedo representaría la transición entre las fases finales del MIS 3 e inicios del MIS 2 en la región de Ambargasta. En AB-2-B las composiciones negativas de $\delta^{13} \mathrm{C}_{\text {car }}$ responderían a eventos más húmedos que rellenan las lagunas con agua enriquecidas en ${ }^{13} \mathrm{C}$ a partir de la cual ocurre la precipitación de carbonatos autigénicos. Otra actividad microbiana-química que explicaría posiblemente parte de las excursiones negativas de $\delta^{13} \mathrm{C}_{\text {carb }}$ en estas secuencias son los procesos de sulfato-reducción vía bacterias anaerobias (Visscher et al., 2000). Según Dupraz y Visscher (2005), en las reacciones de sulfato-reducción los microorganismos oxidan la materia orgánica formando $\mathrm{H}_{2} \mathrm{~S}_{\text {y }} \mathrm{HCO}_{3}$, empobreciendo el CIDT remanente en ${ }^{13} \mathrm{C}$. Sin embargo, como fuera reportado por Valero-Garcés et al. (1999), el intervalo constituido por láminas limosas-orgánicas-yesíferas crenuladas con un espesor de $25 \mathrm{~cm}$ (AB-2-B; Facies 7; $538-476 \mathrm{~cm}$ ) y buen estado de preservación sugeriría que la degradación de la materia orgánica estuvo fundamentalmente dominada por procesos óxicos. Un estudio sobre la geoquímica de los isótopos estables de $\mathrm{O}$ en muestras de agua subterráneas cercanas a la salina (Zanor et al., 2012) evidenció composiciones de $\delta^{18} \mathrm{O}_{\text {agua }}$ que variaron entre -6.70 a $-5.20 \%$ (promedio $=$ $-5.83 \%$ ), valores muy similares a los obtenidos para los valores de $\delta^{18} \mathrm{O}$ en los carbonatos (-5.55 \%). Por otra parte, el crecimiento de morfologías prismáticas de los cristales de yeso formados bajo capa de agua reflejaría temperaturas de cristalización muy altas $\left(\sim 60^{\circ} \mathrm{C}\right.$ : AB-2-B; Facies 7 ; Figura 5; Cody y Cody, 1988). Un régimen muy cálido sujeto a altas tasas de evaporación conduciría al aumento de temperatura en el ambiente acuático y a la precipitación de yesos, condición climática representada por excursiones positivas del $\delta^{18} \mathrm{O}$ carb de los Grupos 5 y 6 (máximo 4.53 \%o). Zanor et al. (2012) encontraron que las composiciones de $\delta^{18} \mathrm{O}_{\text {agua }}$ de las lagunas salinas actuales fluctuaron desde -2.10 hasta $3.45 \%$ (promedio $=0.95 \%$ ). Todas las muestras de las lagunas actuales mostraron un enriquecimiento isotópico con respecto a las concentraciones del agua de lluvia o del agua subterránea de la zona de recarga, indicando que la evaporación es el mecanismo de fraccionamiento fundamental que controla la señal isotópica en los cuerpos de agua. Asimismo, un aumento en el tiempo de residencia en estos ambientes la- 
custres evolucionados pudo haber contribuido al enriquecimiento isotópico del CIDT. Sincrónico al ambiente lacustre central, una planicie fangosa marginal con lagunas efímeras (subfacies 1c) se desarrolló en el borde este de la salina, sugiriendo alimentación de agua subterránea de manera intermitente. Con base en el hallazgo de diferente fauna fósil en las Pampas de Argentina, Tonni et al. (1999) infirieron un cambio a partir de un clima más benigno (30000 años AP) hasta condiciones más áridas (25000 años AP) asociado a una variación en la vegetación, alternando desde ambientes boscosos (herbívoros cursoriales) a pastizales abiertos (grandes pastadores).

Seguidamente, un cambio hacia condiciones más secas interrumpidas por pulsos húmedos se instaló en Ambargasta entre los 23600 y los 18500 años cal. AP (Figura 7). Con el comienzo de esta fase evolutiva, se consideró el inicio del UGM en el área de Ambargasta, concomitante a lo reportado por Kaplan et al. (2008) para el sur de Sudamérica (25000 - 18000 años AP). Tonni et al. (1999) y Prieto (2000) reportaron condiciones más secas para el UGM local ( 21000 años AP) mediante el estudio de fósiles de mamíferos y polen en la región Pampeana de Argentina, respectivamente. AB-2-C (laguna efímera que alterna con una planicie fangosa salina: $476-374 \mathrm{~cm}$ ) exhibió las concentraciones promedio de $\delta^{13} \mathrm{C}_{\text {carb }}$ más negativas del registro de AB-2 indicando aportes de aguas superficiales y subterráneas enriquecidas en ${ }^{12} \mathrm{C}$. El rango más amplio de los valores de $\delta^{13} \mathrm{C}$ carb con respecto a los ambientes de depositación anteriores indicó una gran inestabilidad del reservorio del carbono. Una contribución importante de agua subterránea alimentó las lagunas efímeras mientras que grandes fluctuaciones del nivel freático condujeron a la precipitación de carbonatos intersticiales en ambientes de planicies fangosas salinas. La PPI ${ }_{550}$ y $\delta^{13} \mathrm{C}_{\text {carb }}$ mostraron una correlación negativa altamente significativa $(\mathrm{r}=-0.79)$ evidenciando un alto contenido biológico en las lagunas derivado de diversas fuentes en las etapas de inundación (incluso aporte de material orgánico detrítico). De manera similar, Piovano et al.
(2004) encontraron altas productividades primarias en una laguna salina del centro de Argentina (Laguna Mar Chiquita) durante fases lacustres de nivel alto registradas por valores $\delta^{13} \mathrm{C}_{\text {carb }}$ negativos. En las planicies fangosas capilares de las zonas de borde se desarrollaron cuerpos de agua intermitentes sujetos a la influencia de agua subterránea (Subfacies 1b).

Desde los 18500 a los 8600 años cal. AP, un balance hídrico negativo dominante es inferido a partir del desarrollo de una extensa planicie fangosa salina (AB-2-D) con precipitación de yesos intrasedimentarios rodeada de planicies fangosas capilares (AB-1-A: subfacies 1a; Figura 7). Las fluctuaciones menores de la curva del $\delta^{13} \mathrm{C}_{\text {carb }}$ evidenciaron que el reservorio del carbono no sufrió grandes cambios durante este intervalo de tiempo. Las respuestas de $\delta^{13} \mathrm{C}_{\text {carb }}$ y $\delta^{18} \mathrm{O}_{\text {carb }}$ estarían fundamentalmente controladas por la composición isotópica del nivel freático y por el efecto de una fuerte evaporación que en conjunto propiciarían el crecimiento intrasedimentario de los cristales de yeso. La correlación positiva encontrada entre $\mathrm{PPI}_{1000}$ y $\delta^{13} \mathrm{G}_{\text {carb }}(\mathrm{r}=0.55$; subfacies $1 \mathrm{a})$ avalaría la interpretación de una precipitación carbonática a partir de la fluctuación del nivel freático, donde el agua intersticial pierde $\mathrm{CO}_{2}$ al ascender por intercambio gaseoso. Otras interpretaciones climáticas mediante el uso de secuencias polínicas evidenciaron fases subhúmedas secas a semiáridas durante el Pleistoceno tardío (16000 - 12000 años cal. AP), coetáneo con el Glacial Tardío (Caballero et al., 2010; Denton et al., 2010) en la región Pampeana Argentina (Tonello y Prieto, 2010) y el noroeste argentino (Martini et al., 2013).

Finalmente, las proxies analizadas indicaron un régimen más seco para el Holoceno (8600 - 0 años cal. AP; Figura 7). Durante esta etapa, una expansión de las planicies fangosas capilares con sedimentación siliciclástica dominante (AB-1-A y AB-2-E) indicó una disminución de la precipitación efectiva. Stutz et al. (2012) analizaron polen, palinomorfos no polínicos, macrorestos vegetales y fauna asociada en la laguna Lonkoy en la Llanura Pampeana Argentina y encontraron que desde 
el Holoceno Medio hasta los 2000 años cal. AP existieron fases claras en el ambiente lacustre indicando condiciones más secas que las actuales, fluctuaciones de sequía e inundaciones y/o alta evaporación. De la misma forma, Irurzun et al. (2014) identificó un periodo más seco durante el Holoceno medio y tardío (hasta los 250 años AP), con base en estudios magnéticos en el registro sedimentario de la Laguna La Brava (SE de las Pampas en Argentina). Por su parte, Piovano et al. (2004) identificó un comportamiento hidrológico con balance negativo para el Holoceno medio en la Laguna Mar Chiquita, con un punto de sequía extremo datado en 4200 años cal. AP. Un pulso húmedo de corta duración y de edad desconocida ocurrido durante el Holoceno estuvo representado en Ambargasta por un depósito de limos gruesos ricos en biotitas con valores muy negativos de $\delta^{13} \mathrm{C}_{\text {carb }}(-9.11 \%$ y $-10.69 \%$; Facies 3: AB-1-A y AB-2-E; Figuras 4 y 5). El evento de inundación registrado sería ocasionado por una expansión de los cursos de agua desde la paleo-planicie de inundación del Río Dulce en la zona norte de Ambargasta o de los sectores montañosos del sector este (Sierras Pampeanas; Figura 1). Prado y Alberdi (1999) definieron un episodio cálido y húmedo datado a los ca. 7500 - 5000 mediante restos de comunidades de mamíferos encontrados en la región de las Pampas de Argentina (Óptimo climático del Holoceno? 7600 - 6000 años AP: Clapperton, 1993).

En la actualidad, los ambientes lacustres son intermitentes, someros (30 $\mathrm{cm}$ de profundidad máxima) y se mantienen con agua solamente durante la estación lluviosa (primavera y verano austral; Figura 7). En el presente, la fase evaporítica disponible es la halita, la cual se disuelve con las lluvias y re-precipita en la etapa seca a lo largo de un ciclo anual. El presente estudio paleolimnológico en Ambargasta como así también el análisis de la dinámica ambiental actual no registra el incremento reciente en las precipitaciones desde la década de 1970 el cual no tiene precedentes en el registro instrumental en el subtrópico de Sudamérica (Jacques-Coper y Garreaud, 2014). La colmatación progresiva de este ambiente evaporítico a lo largo del Cuaternario posiblemente contribuyó a disminuir la tasa de acomodación sedimentaria (i.e., Carroll y Bohacs, 1999), limitando la alimentación subterránea y el progreso de ambientes lacustres perennes.

\section{Conclusiones}

1. La reconstrucción paleoambiental en la salina de Ambargasta evidenció una etapa evolutiva más seca durante el periodo $c a .44700$ - 39660 años cal. AP.

2. Con base en la geoquímica de isótopos estables, las inferencias climáticas indicaron que entre los ca. 39600 y 23600 años cal. AP existieron condiciones más húmedas y cálidas de extensa duración en la región de Ambargasta, en correspondencia con el MIS 3. Los valores promedio más negativos de $\delta^{13} \mathrm{G}_{\text {carb }}$ reforzaron las interpretaciones de un importante aporte de agua que permitió el desarrollo de lagunas salinas perennes y efimeras. Las excursiones positivas de $\delta^{18} \mathrm{O}_{\text {carb }}$ avalaron la interpretación de ambientes lacustres sujetos a altas tasas de evaporación que condujeron a la precipitación carbonática y sulfatada en los cuerpos de agua. A partir de los ca. 23600 años cal. AP se consideró el comienzo del registro del UMG en Ambargasta, con base en lo propuesto para el sur de Sudamérica (25000 - 18000 años AP). La expansión de planicies fangosas salinas y capilares en concordancia con valores más positivos de $\delta^{13} \mathrm{C}_{\text {carb }}$ y más negativos de $\delta^{18} \mathrm{O}_{\text {carb }}$ indicaron una disminución en el aporte de agua y una menor evaporación, respectivamente.

3. La ausencia de correlación entre $\delta^{13} \mathrm{C}_{\text {carb }}$ vs. $\delta^{18} \mathrm{O}_{\text {carb }}$ en ambos archivos sedimentarios evidenciaría que el ambiente salino no se mantuvo hidrológicamente cerrado desde los $c a$. 45000 años hasta el presente, ya que probablemente existió salida de agua del sistema o los isótopos no respondieron de manera simultánea a los efectos de la descarga de aguas 
isotópicamente livianas y a los cambios en la tasa de evaporación. Sin embargo, el relleno de la laguna perenne carbonatada mostró una correlación positiva entre $\delta 13 \mathrm{C}_{\text {carb }}$ y $\delta^{18} \mathrm{O}_{\text {carb }}$, indicando un encerramiento hidrológico temporalmente restringido ( $c a .39600-26700$ años cal. AP).

4. Los picos positivos de $\delta^{13} \mathrm{G}_{\text {carb }}$ vs. $\delta^{18} \mathrm{O}_{\text {carb }}$ registrados en los ambientes de lagunas carbonatadas y sulfatadas se explicarían por aumentos en la fotosíntesis acuática y la evaporación durante etapas de concentración evaporítica estacional. Las correlaciones positivas (laguna carbonatada) y negativas (laguna sulfatada) entre $\mathrm{PPI}_{550}$ y $\mathrm{PPI}_{1000}$ fueron indicativas de la paleoproductividad lacustre. En las lagunas carbonatadas la precipitación de carbonatos se asociaría a una mayor productividad primaria mientras que en las lagunas sulfatadas una alta productividad orgánica progresaría en conjunto con el desarrollo de costras de yeso.

5. El registro paleohidrológico de la Salina de Ambargasta reveló que este sistema se encontraría en fase hidrológica con otros archivos paleolimnológicos ubicados al este de la DA, exhibiendo una fase de mayor humedad asociada a un intervalo más cálido. Esta componente húmeda-cálida del Pleistoceno tardío durante el MIS 3 sugirió un aumento generalizado de la precipitación durante este lapso de tiempo en latitudes medias de Argentina, en comparación con las condiciones más secas a partir del UGM (MIS 2 y MIS 1).

6. Otras reconstrucciones paleohidrológicas de baja y alta frecuencia se requieren para correlacionar las tendencias climáticas inferidas en el presente estudio. Es importante conocer la dinámica de la paleocirculación atmosférica en el subtrópico durante el Pleistoceno y Holoceno para perfeccionar el patrón de variabilidad paleoclimática a escala subcontinental en el sur de Sudamérica.

\section{Agradecimientos}

Esta investigación fue financiada por el Consejo Nacional de Investigaciones Científicas y Técnicas (CONICET, Argentina) a través del proyecto PIP 112-200801-00808, por FONCyT-Agencia Nacional de Promoción Científica y Tecnológica (PICT-1371-Raíces; Argentina). Gabriela Zanor agradece al Consejo Nacional de Investigaciones Científicas y Técnicas (CONICET, Argentina) que le otorgó una beca de doctorado y a la Federal Commission for Scholarships for Foreign Students (DHA/OFES, Suiza) por la beca otorgada para su estancia en la Universidad de Ginebra.

\section{Referencias}

Álvarez, L.A, Fernández Seveso, F., Pérez, M.A., Bolatti, N.D., 1990, Estratigrafía de la Cuenca Saliniana (resumen), en XI Congreso Geológico Argentino: San Juan, Argentina, Asociación Geológica Argentina, 145-148.

Bard, E., 1998, Geochemical and geophysical implications of the radiocarbon calibration: Geochimica et Cosmochimica Acta, 62, 2025-2038.

Blasi, A., Castiñeira Latorre, C., Del Puerto, L., Prieto, A.R., Fucks, E., De Francesco, C., Young, A., 2010, Paleoambientes de la cuenca media del río Luján (Buenos Aires, Argentina) durante el último período glacial (EIO 4-2): Latin American Journal of Sedimentology and Basin Analysis, 17(2), 85-111.

Bruniard, E., 1982, La diagonal árida Argentina: un límite climático real: Revista Geográfica, 95, 5-20.

Caballero, M., Lozano-García, S., VázquezSelem, L., Ortega, B., 2010, Evidencias de cambio climático y ambiental en registros glaciales y cuencas lacustres del centro de México durante el Último Máximo Glacial: Boletín de la Sociedad Geológica Mexicana, 62(3), 359-377. 
Canfield, D.E., Sorensen, K.B., Oren, A., 2004, Biochemistry of a gypsum-encrusted microbial ecosystem: Geobiology, 2, 133-150.

Carroll, A.R., Bohacs, K.M., 1999, Stratigraphic classification of ancient lakes: Balancing tectonic and climatic controls: Geology, 27, 99-102.

Chivas, A.R., De Dekker, P., Cali, J.A., Chapman, A., Kiss, E., Shelly, J.M.G., 1993, Coupled stable isotope and trace element measurements of lacustrine carbonates as paleoclimatic indicators, en Swart, P.K., Lohmann, K.C., McKenzie, J.A., Savin, S. (eds.), Climate Change in Continental Isotopic Records: United States, American Geophysical Union, 113-122.

Clapperton, Ch.M., 1993, Nature of environmental changes in South America at the Last Glacial Maximum: Palaeogeography, Palaeoclimatology, Palaeoecology, 101, 189-208.

Cody, R.D., Cody, A.M., 1988, Gypsum nucleation and crystal morphology in analog saline terrestrial environments: Journal of Sedimentary Petrology, 58, 247-255.

Cohen, A., 2003, Paleolimnology: The History and Evolution of Lake Systems: New York, Oxford University Press, 500 p.

Córdoba, F.E., Guerra, L., Cuña Rodríguez, C., Sylvestre, F., Piovano, E.L., 2015, Una visión paleolimnológica de la variabilidad hidroclimática reciente en el centro de argentina: desde la pequeña edad de hielo al siglo XXI: Latin American Journal of Sedimentology and Basin Analysis, 21, 65-75.

Costa, K., Russell, J., Konecky, B., Lamb, H., 2014, Isotopic reconstruction of the African Humid Period and Congo Air Boundary migration at Lake Tana, Ethiopia: Quaternary Science reviews, 83, 58-67.

Dargám, R.M., 1995, Geochemistry of waters and brines from the Salinas Grandes basin,
Cordoba, Argentina, I. Geomorphology and hydrochemical caracteristics: International Journal of Salt Lake Research, 3, 137-158.

Dargám, R.M., Depetris, P.J., 1995, Mecanismos de control hidroquímico en aguas y salmueras de las Salinas Grandes, Provincia de Córdoba: Revista de la Asociación Geológica Argentina, 50, 87-102.

Dávila, F.M., Astini, R.A., 2003, Early Middle Miocene broken foreland development in the southern Central Andes: evidence for extension prior to regional shortening: Basin Research, 15, 379-396.

Denton, G.H., Anderson, R.F., Toggweiler, J.R., Edwards, R.L., Schaefer, J.M., Putnam, A.E., 2010, The last glacial termination: Science, $328,1652-1656$.

Dupraz, G., Visscher, P.T., 2005, Microbial lithification in marine stromatolites and hypersaline mats: Trends in Microbiology, 13, 429-438.

Gasparini, G.M., Rabassa, J., Deschamps, C., Tonni, E.P., 2016, Marine Isotope Stage 3 in Southern South America, 60 KA B.P.-30 KA B.P.: Switzerland, Springer International Publishing, $354 \mathrm{p}$.

Garreaud, R.D., Vuille, M., Compagnucci, R., Marengo, J., 2009, Present-day South American Climate (LOTRED South America): Palaeogeography, Palaeoclimatology, Palaeoecology, 281, 180-195.

Gilli, A., Ariztegui, D., Anselmetti, F.S., McKenzie, J.A., Markgraf, V., Hajdas, I., McGulloch, R.D., 2005, Mid-Holocene strengthening of the Southern Westerlies in South America - Sedimentological evidences from Lago Cardiel, Argentina $\left(49^{\circ} \mathrm{S}\right)$ : Global and Planetary Change, 49, 75-93.

Grodsky, S.A., Carton, J.A., 2003, The Intertropical Convergence Zone in the South Atlantic and the Equatorial cold tongue: Journal of Climate, 16, 723-733. 
Guerra, L., Piovano, E., Córdoba, F.E., Sylvestre, F., Damatto, S., 2015, The hydrological and environmental evolution of shallow Lake Melincué, central Argentinean Pampas, during the last millennium: Journal of Hydrology, 529, 570-583.

Heiri, O., Lotter, A. F., Lemcke, G., 2001, Loss on ignition as a method for estimating organic and carbonate content in sediments: reproducibility and comparability of results: Journal of Paleolimnology, 25, 101-110.

Irurzun, M.A., Gogorza, C.S., Sinito, A.M., Chaparro, M.A.E., Prieto, A.R., Laprida, C., Lirio, J.M., Navas, A.M., Nuñez, H., 2014, A high-resolution palaeoclimate record for the last 4800 years from lake La Brava, SE Pampas plains, Argentina: Geofísica Internacional, 53, 365-383.

Jacques-Coper, M., Garreaud, R., 2014, Characterization of the 1970s climate shift in South America: International Journal of Climatology, 35, 2164-2179.

Jones, B.F., Deocampo, D.M., 2003, Geochemistry of Saline Lakes, en Drever, J.I. (ed.), Surface and Ground Water, Weathering and Soils, Treatise on Geochemistry: New York, Elsevier, 393-424.

Jordan, T.E., Allmendinger, R.W., 1986, The Sierras Pampeanas of Argentina: a modern analogue of Rocky Mountain foreland deformation: American Journal of Science, 286, 737-764.

Kaplan, M.R., Fogwill, G.J., Sugden, D.E., Hulton, N.R.J., Kubik, P.W., Freeman, S.P.H.T., 2008, Southern Patagonian glacial chronology for the Last Glacial period and implications for Southern Ocean climate: Quaternary Science Reviews, 27, 284-294.

Kröhling, D.M., Iriondo, M., 1999, Upper Quaternary palaeoclimates of the Mar Chiquita area, North Pampa, Argentina: Quaternary International, 57, 149-163.
Kull, C., Francine Hänni, F., Grosjean, M., Veit, H., 2003, Evidence of an LGM cooling in NW-Argentina (221S) derived from a glacier climate model: Quaternary International, 108, 3-11.

Lamb, A.L., Leng, M.J., Lamb, H.F., Mohammed, M.U., 2000, A 10000-year oxygen and carbon isotope record of hydrological change in a small Ethiopian crater lake: The Holocene, 10, 167-77.

Lamb, A.L., Leng, M.J., Lamb, H.F, Telford, R.J., Mohammed, M.U., 2002, Climatic and non-climatic effects on the $\delta^{18} \mathrm{O}$ and $\delta^{13} \mathrm{C}$ compositions of Lake Awassa, Ethiopia, during the last $6.5 \mathrm{ka}$ : Quaternary Science Reviews, 21, 2199-2211.

Leng, M.J., Marshall, J.D., 2004, Paleoclimate interpretation of stable isotope data from lake sediment archives: Quaternary Science Reviews, 23, 811-831.

Leng, M.J., Lamb, A.L., Marshall, J.D., Wolfe, B.B., Jones, M.D., Holmes, J.A., Arrowsmith, C., 2005, en Leng, M.J. (ed.), Isotopes in Palaeoenvironmental Research: Netherlands, Springer, 147-184.

Li, H., Ku, T., 1997, $\delta^{13} \mathrm{C}-\delta^{18} \mathrm{O}$ covariance as paleohydrological indicator for closed-basin lakes: Paleogeography, Paleoclimatology, Paleoecology, 133, 69-80.

Liebmann, B., Kiladis, G.N., Marengo, J.A., Ambrizzi, T., Glick, J.D., 1999, Submonthly convective variability over South America and the South Atlantic convergence zone: Journal of Climate, 12, 1977-1991.

Lira, R.H., Millone, H.A., Kirschbaum A.M., Moreno, R.S., 1997, Calc-Alkaline arc granitoid in the Sierra Norte-Ambargasta Ranges, central Argentina: Journal of South American Earth Sciences, 10, 157-177.

Liu, W., Li, X., Zhang, L., An, Z., Xu, L., 2009, Evaluation of oxygen isotopes in carbonate as an indicator of lake evolution in arid areas: The modern Qinghai Lake, Qinghai-Tibet Plateau: Chemical Geology, 268, 126-136. 
Lucero Michaut, N., 1979, Sierras Pampeanas del norte de Córdoba, sur de Santiago del Estero, borde oriental de Catamarca y ángulo sudeste de Tucumán, en Segundo Simposio de Geología Regional Argentina: Córdoba, Argentina, Academia Nacional de Ciencias, 293-348.

Martini, M.A., Strelin, J.A., Astini, R.A., 2013, Inventario y caracterización morfoclimática de los glaciares de roca en la Cordillera Oriental argentina (entre $22^{\circ}$ y $25^{\circ} \mathrm{S}$ ): Revista Mexicana de Ciencias Geológicas, 30(3), 569-581.

McKenzie, J.A., Hollander, D.J., 1993, OxygenIsotope Record in Recent Carbonate Sediments from Lake Greifen, Switzerland (1750-1986), en Swart, P.K., Lohmann, K.C., McKenzie, J.A., Savin, S. (eds.), Climate Change in Continental Isotopic Records: United States, American Geophysical Union, 101-112.

Mayr, C., Fey, M., Haberzettl, T., Janssen, S., Lücke, A., Maidana, N., Ohlendorf, C., Schäbitz, F., Schleser, G., Wille, M., Zolitschka, B., 2005, Paleoenvironmental changes in southern Patagonia during the last millennium recorded in lake sediments from Laguna Azul (Argentina): Palaeogeography, Palaeoclimatology, Palaeoecology 228, 203-227.

Nogués-Paegle, J., Mo, K.C., 1997, Alternating wet and dry conditions over South America during summer: Monthly Weather Review, 125, 279-291.

Piovano, E., Ariztegui, D., Bernasconi, S.M., McKenzie, J.A., 2004, Stable isotopic record of hydrological changes in subtropical Laguna Mar Chiquita (Argentina) over the last 230 years: The Holocene, 14, 525-535.

Piovano, E., Ariztegui, D., Córdoba, F., Cioccale, M., Sylvestre, F., 2009, Hydrological variability in South America below the Tropic of Capricorn (Pampas and eastern Patagonia, Argentina) during the last 13.0 ka., en Vimeux, F., Sylvestre, F., Khodri, M. (eds.), Past climate variability from the Last Glacial Maximum to the Holocene in South America and Surrounding regions: From the Last Glacial Maximum to the Holocene: Dordrecht, Springer, 323-351.

Prado, J.L, Alberdi, M.T., 1999, The mammalian record and climatic change over the last 30000 years in the Pampean Region, Argentina: Quaternary International, 57-58, $165-174$.

Prieto, A.R., 2000, Vegetational history of the Late Glacial-Holocene transition in the grasslands of eastern Argentina: Palaeogeography, Palaeoclimatology, Palaeoecology, 157, 167-188.

Quattrocchio, M.E., Borromei, A.M., Deschamps,C.M., Grill, S.C., Zavala, C.A, 2008, Landscape evolution and climate changes in the Late Pleistocene-Holocene, southern Pampa (Argentina): Evidence from palynology, mammals and sedimentology: Quaternary International, 181, 123-138.

Rabassa, J., Ponce, J.F., 2013, The Heinrich and Dansgaard-Oeschger climatic events during Marine Isotopic Stage 3: Searching for appropriate times for human colonization of the Americas: Quaternary International, 299, 94-105.

Ramos, V.A., Cristallini, E.O., Pérez, D.J., 2002, The Pampean flat-slab of the central Andes: Journal of South American Earth Sciences, 15, 59-78.

Rothschild, L.J., Giver, L.J., White, M.R., Mancinelli, R.L., 1994, Metabolic activity of microorganisms in evaporates: Journal of Phycology, 30, 431-438.

Rutter, N., Coronato, A., Helmens, K., Rabassa, J., Zárate, M., 2012, The Glacial and Loess Record of Southern South America, en Hamilton, K., Lohmann, G., Mysak, L.A., Rabassa, J. (eds.), Glaciations in North and South America from the Miocene to the Last Glacial Maximum: Netherlands, Springer, $1-23$. 
Saulo, A.C., Nicolini, M., Chou, S.C., 2000, Model characterization of the South American low-level flow during the 1997-98 spring-summer season: Climate Dynamics, 16, 867-881.

Seluchi, M.E., Marengo, J.A., 2000, Tropicalmidlatitude exchange of air masses during summer and winter in South America: climatic aspects and examples of intense events: International Journal of Climatology, 20, 1167-1190.

Spötl, G., Vennemann, T.W., 2003, Continuousflow IRMS analysis of carbonate minerals: Rapid Communications in Mass Spectrometry, 17, 1004-1006.

Statgraphics Plus Ver. 5.1 Professional, 2001, STSC and Statistical Graphics Corporation: Maryland, USA, Bekersville, Programa informativo, 1 CD ROOM.

Steinman, B.A., Abbott, M.B., 2013, Isotopic and hydrologic responses of small, closed lakes to climate variability: Hydroclimate reconstructions from lake sediment oxygen isotope records and mass balance models: Geochimica et Cosmochimica Acta, 105, 342-359.

Stutz, S., Borel, M., Fontana, S., del Puerto, L., Inda, H., García-Rodríguez, F., Tonello, M, 2010, Late Holocene climate and environment of the SE Pampa grasslands, Argentina, inferred from biological indicators in shallow, freshwater Lake Nahuel Rucá: Journal of Paleolimnology, 44, 761-775.

Stutz, S., Borel, G.M., Fontana, S.L.,. Tonello, M.S, 2012, Holocene changes in trophic states of shallow lakes from the Pampa plain of Argentina: The Holocene 22, 1263-1270.

Talbot, M.R., 1990, A review of the paleohydrological interpretation of carbon and oxygen isotopic ratios in primary lacustrine carbonates: Chemical Geology, 80, 261-279.
Talbot, M.R., Kelts, K., 1990, Paleolimnological signatures from carbon and oxygen isotopic ratios in primary lacustrine sediments, en Katz, B. (ed.), Lacustrine basin exploration: Case Studies and Modern Analogs: United States, American Association of Petroleum Geologists Memoir, 99-112.

Tonello, M.S., Prieto, A.R., 2010, Tendencias climáticas para los pastizales pampeanos durante el Pleistoceno tardío-Holoceno: Estimaciones cuantitativas basadas en secuencias polínicas fósiles partir de registros polínicos fósiles: Ameghiniana, 47, 501-514.

Tonni, E.P., Cione, A.L., Figini, A.J., 1999, Predominance of arid climates indicated by mammals in the pampas of Argentina during the Late Pleistocene and Holocene: Palaeogeography, Palaeoclimatology, Palaeoecology, 147, 257-281.

Valero-Garcés, B.L., Grosjean, M., Kelts, K., Schreier, H., Messerli, B., 1999, Holocene lacustrine deposition in the Atacama Altiplano: facies models, climate and tectonic forcing: Palaeogeography, Palaeoclimatology, Palaeoecology, 151, 101-125.

Valero-Garcés, B.L., Delgado-Huertas, A., Navas, A., Machín,J., González-Sampériz, P., Kelts, K., 2000, Quaternary palaeohydrological evolution of a playa lake: Salada Mediana, central Ebro Basin, Spain: Sedimentology, 47, 1135-1156.

Valero-Garcés, B.L., Delgado-Huertas, A., Navas, A., Edwards, L., Schwalb, A., Ratto, N., 2003, Patterns of regional hydrological variability in central southern Altiplano $\left(18^{\circ}-26^{\circ} \mathrm{S}\right)$ lakes during the last 500 years: Palaeogeography, Palaeoclimatology, Palaeoecology, 194, 319-338.

Vera, C., Higgins, W., Amador, J., Ambrizzi, T., Garreaud, R., Gochis, D., Gutzler, D., Lettenmaier, D., Marengo, J., Mechoso, C., Nogués-Paegle, J., Silva Diaz, P.L., Zhang, C., 2006, Towards a unified view of the American Monsoon System: Journal of Climate, 19, 4977-5000. 
Visscher, P.T., Reid, R.P., Bebout, B.M., 2000, Microescale observations of sulfate reduction: Correlation of microbial activity with lithified micritic laminae in modern marine stromatolites: Geology, 28, 919-922.

Visscher, P.T., Stolz, J.F., 2005, Microbial mats as bioreactors: populations, processes and products: Palaeogeography, Palaeoclimatology, Palaeoecology, 219, 87-100.

Wright, J.D., 2000, Global climate change in marine stable isotope records, en Noller, J.S., Sowers, J.M., Lettis, W.R. (eds.), Quaternary Geochronology, Methods and Applications: AGU Reference Shelf, American Geophysical Union, 4, 427-433.

Zanor, G., Piovano, E., Ariztegui, D., ValletCoulomb, G., 2012, A modern subtropical playa complex: Salina de Ambargasta, central Argentina: Journal of South American Earth Sciences, 35, 10-26.
Zanor, G.A., Piovano, E.L., Ariztegui, D., Pasquini, A.I., Chiesa, J.O., 2013, El registro sedimentario Pleistoceno tardío-Holoceno de la Salina de Ambargasta (Argentina central): una aproximación paleolimnológica: Revista Mexicana de Ciencias Geológicas, 30, 336-354.

Zárate, M., 2003, Loess of southern South America: Quaternary Science Reviews, 22, 1987-2006.

Zárate, M.A., Tripaldi, A., 2012, The aeolian system of central Argentina: Aeolian Research, 3, 401-417.

Zhou, J., Lau, K.M., 1998, Does a monsoon climate exist over South America?: Journal of Climate, 11, 1020-1040. 